\title{
CANDELSz7: a large spectroscopic survey of CANDELS galaxies in the reionization epoch
}

\author{
L. Pentericci ${ }^{1}$, E. Vanzella ${ }^{2}$, M. Castellano ${ }^{1}$, A. Fontana ${ }^{1}$, S. De Barros ${ }^{3}$, A. Grazian ${ }^{1}$, F. Marchi ${ }^{1}$, M. Bradac ${ }^{4}$, \\ C. J. Conselice ${ }^{5}$, S. Cristiani ${ }^{6}$, M. Dickinson ${ }^{7}$, S. L. Finkelstein ${ }^{8}$, E. Giallongo ${ }^{1}$, L. Guaita ${ }^{1,9}$, A. M. Koekemoer ${ }^{10}$, \\ R. Maiolino ${ }^{11}$, P. Santini ${ }^{1}$, and V. Tilvi ${ }^{2}$
}

1 INAF, Osservatorio Astronomico di Roma, via Frascati 33, 00078 Monteporzio Catone, Italy e-mail: laura.pentericci@oa-roma.inaf.it

2 INAF, Osservatorio Astronomico di Bologna, via Gobetti 93/3, 40129 Bologna, Italy

3 Observatoire de Genève, Université de Genève, $51 \mathrm{Ch}$. des Maillettes, 1290 Versoix, Switzerland

${ }^{4}$ Department of Physics, University of California, 1 Shields Ave, Davis, CA 95616, USA

5 School of Physics \& Astronomy, The University of Nottingham, University Park, Nottingham NG7 2RD, UK

${ }^{6}$ INAF-Osservatorio Astronomico di Trieste, via Tiepolo 11, 34143 Trieste, Italy

7 NOAO, 959 N. Cherry Ave, Tucson, AZ 85719, USA

8 Department of Astronomy, The University of Texas at Austin, Austin, TX 78712, USA

9 Núcleo de Astronomía, Facultad de Ingeniería y Ciencias, Universidad Diego Portales, Av. Ejército 441, Santiago, Chile

10 Space Telescope Science Institute, 3700 San Martin Drive, Baltimore, MD 21218, USA

11 Kavli Institute for Cosmology, University of Cambridge, Madingley Road, Cambridge CB3 OHA, UK

12 School of Earth \& Space Exploration, Arizona State University, Tempe, AZ 85287, USA

Received 15 December 2017 / Accepted 3 August 2018

\begin{abstract}
We present the results of CANDELSz7, a European Southern Observatory (ESO) Large Program aimed at spectroscopically confirming a homogeneous sample of $z \simeq 6$ and $z \simeq 7$ star forming galaxies. The candidates were selected in the GOODS-South, UDS, and COSMOS fields using the official CANDELS catalogs based on $H_{160}$-band detections. Standard color criteria, which were tailored depending on the ancillary multi-wavelength data available for each field, were applied to select more than 160 candidate galaxies at $z \simeq 6$ and $z \simeq 7$. Deep, medium-resolution FORS2 spectroscopic observations were then conducted with integration times ranging from 12 to $20 \mathrm{~h}$ to reach a Ly $\alpha$ flux limit of approximately $1-3 \times 10^{-18} \mathrm{erg} \mathrm{s}^{-1} \mathrm{~cm}^{-2}$ at $3 \sigma$. We could determine a spectroscopic redshift for about $40 \%$ of the galaxies, mainly through the detection of a single emission line that we interpret as Ly $\alpha$ emission, or for some of the brightest objects $\left(H_{160} \leq 25.5\right)$ from the presence of faint continuum and a sharp drop that we interpret as a Ly $\alpha$ break. In this paper we present the redshifts and main properties of 65 newly confirmed high-redshift galaxies. Adding previous proprietary and archival data we assemble a sample of $\simeq 260$ galaxies that we use to explore the evolution of the Ly $\alpha$ fraction in Lyman break galaxies and the change in the shape of the emission line between $z \sim 6$ and $z \sim 7$. We also discuss the accuracy of the CANDELS photometric redshifts in this redshift range.
\end{abstract}

Key words. galaxies: high-redshift - galaxies: distances and redshifts - dark ages, reionization, first stars

\section{Introduction}

The exploration of the reionization era is surely one of the most challenging and fascinating tasks of present-day extragalactic astronomy. For the first time we can compare precise results from cosmic microwave background data from Planck (Planck Collaboration Int. XLVII 2016) to observations of primeval galaxies, when the Universe was still largely neutral. These observations help us to understand the exact time-line of the reionization process and how it proceeded spatially, and to identify the sources that produced all or most of the ionizing photon budget. The general consensus seems to be that galaxies, and in particular the faintest systems, were those providing most of the ionizing radiation (e.g., Bouwens et al. 2016; Finkelstein et al. 2015), although faint active galactic nuclei (AGN) might also have played a role (e.g., Giallongo et al. 2015).

To understand the evolution of the reionization process, one of the key quantities we would like to measure is the fraction of neutral hydrogen present in the Universe and its evolution with cosmic time. In the future, the Square Kilometer Array (SKA) and maybe its precursors will be able to directly detect the neutral hydrogen content in the early Universe by mapping the $21 \mathrm{~cm}$ emission. In the meantime we must rely on alternative observational probes, which allow us to set indirect constraints on the amount of neutral hydrogen. These include deep optical spectra of high-redshift quasi-stellar objects (QSO) where we can analyze the Gunn-Peterson optical depth (e.g., Fan et al. 2006), the distribution of dark gaps (Chardin et al. 2018; McGreer et al. 2015), the analysis of damping absorption wings, as in Schroeder et al. (2013), and the analysis of gamma-ray burst (GRB) spectra (Totani et al. 2014). For Lyman break galaxies (LBGs) and Ly $\alpha$ emitters (LAEs) the most promising tools are studying the prevalence of Ly $\alpha$ emission in star-forming galaxies (Pentericci et al. 2014, hereafter LP14; Pentericci et al. 2011, hereafter LP11; Ono et al. 2012; Treu et al. 2013; Schenker et al. 2014; Caruana et al. 2014; 
Tilvi et al. 2014), studying the evolution of the clustering, and studying the luminosity function of LAEs (Ouchi et al. 2010; Tilvi et al. 2010; Sobacchi \& Mesinger 2015).

In particular, several groups have focused their attention on the presence of the Ly $\alpha$ line in samples of LBGs. While from redshift $\sim 2$ to $\sim 6$ the fraction of galaxies showing a bright Ly $\alpha$ emission line seems to increase steadily (Stark et al. 2010; Cassata et al. 2015), there is a strong deficit of such lines in galaxies as we approach $z \sim 7$ (LP14; Tilvi et al. 2014; Treu et al. 2013; Ono et al. 2012; Schenker et al. 2012; LP11; Fontana et al. 2010). This is so far one of the strongest and perhaps the most solid pieces of evidence that at $z \sim 7$ the Universe is partially neutral, since neutral hydrogen can easily suppress the visibility of the line. It would be much harder to explain the observed drop in the $\operatorname{Ly} \alpha$ fraction with a very rapid change in the physical properties of galaxies, such as the intrinsic dust content. The only alternative viable explanation is a sudden increase of the escape fraction of Lyman continuum photons (Mesinger et al. 2015); however despite the recent progress in the discovery of real Lyman continuum emitters (Shapley et al. 2016; Izotov et al. 2016; Vanzella et al. 2016), this quantity remains elusive and assessing its evolution in the early Universe is extremely difficult.

The dramatic decrease of $\operatorname{Ly} \alpha$ fraction at high redshift implies that the number of spectroscopically confirmed galaxies above $z=6.5$ is still very low. This emission line is at present one of the few possible redshift indicators in the reionization epoch, although the Carbon line emission is becoming a viable alternative, from transitions visible in the sub-mm (the [CII] $158 \mu \mathrm{m}$ line e.g., Pentericci et al. 2016; Bradač et al. 2017; Smit et al. 2018), or in the ultraviolet (UV) domain (the CIII]1909 Å emission line, Stark et al. 2015, 2017; Le Fèvre et al. 2017; Maseda et al. 2017). All of the above results are based on small datasets, and statistical fluctuations can be very large. This is particularly true for the faintest LBGs, since most previous observations focused on the brighter candidates $\left(M_{\mathrm{UV}}<-20.5\right)$. The results also came from very heterogeneous observational efforts, in terms of wavelength coverage, sample detection and selection, integration times, and so on; it is therefore hard to combine them and to assess their global statistical significance. To overcome these problems, in 2013 we started an ESO Large Program with FORS2 (Program ID 190.A-0685) to assemble observations of a much larger and homogeneously selected sample of galaxies at $z \sim 6$ and $\sim 7$, to place much firmer constraints on the decrease in the visibility of $\operatorname{Ly} \alpha$ emission between these two epochs, and to assess if and how this decrease depends on galaxies' brightness. This is of particular relevance since the visibility of Ly $\alpha$-emitting galaxies during the Epoch of Reionization is controlled by both diffuse HI patches in large-scale bubble morphology and smallscale absorbers. Improved constraints on the relevant importance of these two regimes can be obtained by analyzing the full UV-luminosity-dependent redshift evolution of the Ly $\alpha$ fraction of Lyman break galaxies (Kakiichi et al. 2016).

In this paper we present the observations and the results of our large program, while deferring a full analysis of the properties of the galaxies and the constraints on reionization models to other papers. In Sect. 2 we describe the target selection, in Sect. 3 we present the observations and data reduction procedure, in Sect. 4 we present the results, and in Sect. 5 we discuss the observational properties of the detected galaxies. In a companion paper (De Barros et al. 2017), we have discussed the physical properties of the "redshift 6" sample, while in Castellano et al. (2017) we investigated the nature of the GOODS-South and

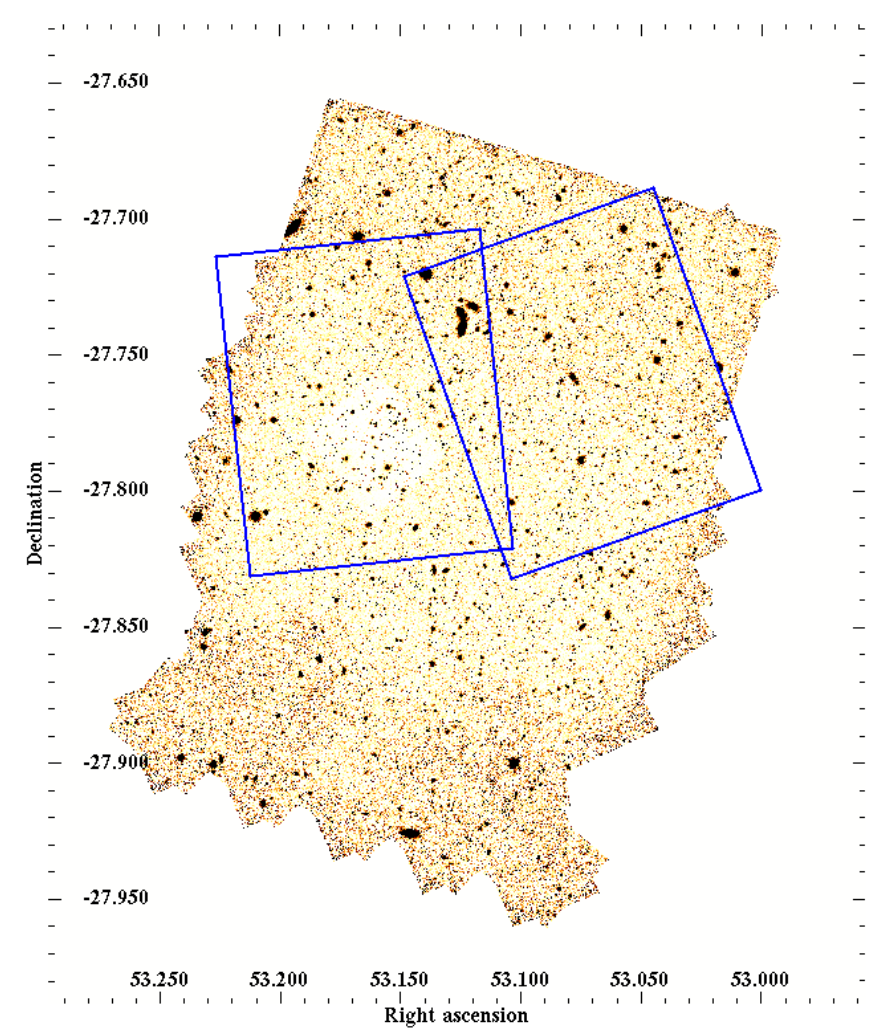

Fig. 1. Layout of the two FORS2 masks in the GOODS south field overplotted on the CANDELS $H_{160}$ image.

UDS $z \sim 7$ target to probe possible physical differences between Ly $\alpha$-emitting and non-emitting sources. Finally, in an upcoming work we will discuss the implication of our results for the reionization epoch more extensively.

We adopt a $\Lambda$-CDM cosmological model with $H_{0}=$ $70 \mathrm{~km} \mathrm{~s}^{-1} \mathrm{Mpc}^{-1}, \Omega_{\mathrm{m}}=0.3$ and $\Omega_{\Lambda}=0.7$. All magnitudes are expressed in the AB system (Oke \& Gunn 1983).

\section{Target selection}

Targets were selected from the CANDELS survey (Grogin et al. 2011; Koekemoer et al. 2011) using the official catalogs in the UDS, GOODS-South, and COSMOS fields (Galametz et al. 2013; Guo et al. 2013; Nayyeri et al. 2017, respectively for the three fields). The detection of the CANDELS catalog is always based on the $H_{160}$-band which is the reddest band available. Most notably at redshift 6 and 7, this band is not affected by the presence of Ly $\alpha$ emission. This minimizes the possibility of bias towards strong line emitters at any redshift. At variance with this, in the pre-CANDELS epoch, $i$-dropouts were typically selected from catalogs where the reddest detection band was the $z$-band (Stark et al. 2010; Vanzella et al. 2008). This meant that in the presence of a strong line emission, fainter galaxies at high redshift were only detected because of the brightening of the measured $z^{\prime}$ magnitude due to the line flux, and this was only partially compensated by the fact that galaxies in the lower part of the redshift selection window would fall out of the selection (see a detailed discussion in Stanway et al. 2007) because their $i-z$ color would not be red enough to pass the cut. In our case, the selection of galaxies in the $H_{160}$-band of course means that amongst objects with similar luminosity at $1500 \AA$ rest-frame, those with a particularly blue slope could 
potentially fall out of detection. However this bias is probably similar at both $z \sim 6$ and 7 since the UV slopes of galaxies do not change appreciably between these two epochs in the magnitude range considered (e.g., Bouwens et al. 2014). In addition to the CANDELS data, $\left(J_{125}\right.$ and $H_{160}$ bands plus $V_{606}$ and $I_{814}$ that are available for all three fields), each of the fields has multiwavelength supporting observations both from ground instruments and from space (see the above papers for a detailed list). In particular, the GOODS-South field has the best optical multiwavelength data, with deep HST imaging available in many different bands, and both UDS and GOODS-South have supporting near-IR data (including deep HAWK-I $K$-band imaging) coming from the HUGS survey (Fontana et al. 2014). The different supporting data result in slightly different selection criteria in each field, although we attempted to apply selection criteria that were as uniform as possible for all fields. The targets for our program were selected in the following way.

(1) For the $z \sim 7$ samples, we employed the color criteria, described in detail in Grazian et al. (2012), for the GOODSSouth field and the ERS sub-region (which has observations in the $Y_{098}$ filter instead of the $Y_{105}$, therefore the criteria are slightly tailored to account for the difference in the transmission) separately, and are

$z-Y_{105}>0.8$

$z-Y_{105}>0.9+0.75\left(Y_{105}-J_{125}\right)$,

$z-Y_{105}>-1.1+4.0\left(Y_{105}-J_{125}\right)$,

for the GOODS-South field, and

$z-Y_{098}>1.1$,

$z-Y_{098}>0.55+1.25\left(Y_{098}-J_{125}\right)$,

$z-Y_{098}>-0.5+2.0\left(Y_{098}-J_{125}\right)$,

for the ERS area. For the non-detection in photometric bands bluer than $Z$, we adopt the same criteria used in Castellano et al. (2010a,b) and in Grazian et al. (2012) which are a signal-tonoise ratio $(\mathrm{S} / \mathrm{N})$ of $S / N<2$ in all BVI HST bands and $S / N<1$ in at least two of them.

For the UDS and COSMOS fields, where the only photometry available from space is in $V_{606}, I_{814}, J_{125}$, and $H_{160}$ bands, we adopt an $I_{814}$-dropout color described also in Grazian et al. (2012), which gives a more extended redshift window for selecting galaxy candidates $(6.4<z<8.5)$ and is the following.

$I_{814}-J_{125}>2.0$,

$I_{814}-J_{125}>1.4+2.5\left(J_{125}-H_{160}\right)$.

In this case, a stricter non-detection was required in the only HST band bluer of the Lyman break available, that is, $S / N\left(V_{606}\right)<$ 1.5 , and non-detections $(S / N<2)$ in all ground-based images bluer than the $R$-band. The redshift selection functions for these different color criteria adopted are shown in Fig. 1 of Grazian et al. (2012).

(2) For the $z \sim 6$ sample in the GOODS-South field we have employed a selection that is similar (but not identical) to the one used in Bouwens et al. (2015): $i_{775}-z_{850}>1.0 \wedge Y_{105}-H_{160}<$ 0.5 , with a requirement for a non-detection in either the $B$ or $V$ band as $\left(S / N\left(B_{435}\right)<2 \wedge V_{606}-z_{850}>2.7\right) \vee S / N\left(V_{606}\right)<2$.

For the COSMOS and UDS fields we used the following criteria: $(i-z)>1.0 \wedge\left(J_{125}-H_{160}\right)<0.5$ with a requirement for non-detection in both the $U$ and $B$ bands as $S / N(U B)<2$.

(3) In addition we also selected galaxies that were not compliant with the above color criteria, but had a photometric redshift between 5.5 and 7.3, that is, the approximate range where we can expect to detect the Ly $\alpha$ emission with the adopted observational setup. The selection with photometric redshift was done to complement (1) and (2) above, since it can recover objects that are scattered out of the color criteria, because of uncertain photometry or because of the presence of the Ly $\alpha$ emission line.

The photometric redshifts adopted were the official CANDELS best redshifts presented in Santini et al. (2015) for the GOODS-South and UDS fields, and by Nayyeri et al. (2017) for the COSMOS field. Briefly, they are based on a hierarchical Bayesian approach that combines the full probability distribution functions $\operatorname{PDF}(z)$ of individual redshift determination provided by several different CANDELS photo- $z$ investigators. The precise techniques adopted to derive the official CANDELS photometric redshifts, as well as the individual values from the various participants, are described in detail by Dahlen et al. (2013) and in the above papers.

Finally all candidates selected in the above categories were visually inspected in all photometric bands available to remove possible false detections due to residual defects in regions of poorer imaging quality. The blue bands were also smoothed to ensure that the non-detection was solid. In addition, galaxies which could plausibly be at high redshift but had very close objects (angular separation $\leq 1-2^{\prime \prime}$ ) that could hamper their spectroscopic identification were also removed from the samples.

We designed three masks for each of the COSMOS and UDS fields and two masks for GOODS-South. The masks were designed to allocate the largest number of $z \sim 7$ galaxies as first priority, and then in order of decreasing priority, $z \sim 6$ candidates, AGN candidates (i.e., X-ray and Herschel sources), and other fillers. The mask construction was driven only by geometrical constraints without any other bias. In each mask we also reserved a number of slits (two per FORS2 chip) to observe bright objects: this is required since our objects (with the exception of some AGN and fillers) were not visible in individual $20 \mathrm{~min}$ exposures and having bright objects was useful to check centering and trace the spectra. Typically, each mask contained between 30 and 40 useful targets. The total number of objects placed in each mask divided by category is reported in Table 1. In Figs. 1-3, we show the position of all FORS2 masks over-laid on the CANDELS $H_{160}$-band images for the GOODS-South, UDS, and COSMOS, respectively. In Fig. 4 we show the color-color selection diagrams for all the $z \sim 7$ candidates inserted in the masks, for the different fields and areas. The density of observed candidates at $z \sim 7$ is similar for the UDS and COSMOS fields $\left(\sim 0.20-0.22 \mathrm{arcmin}^{-2}\right)$ while it is higher for GOODS-South $\left(0.42 \mathrm{arcmin}^{-2}\right)$ because of the increased depth of the CANDELS detection catalog.

\section{Observations and data reduction}

Observations were taken with the FORS2 spectrograph on the ESO Very Large Telescope. We used the $600 \mathrm{Z}$ holographic grating, which provides the highest sensitivity in the range 8000-10000 $\AA$ with a spectral resolution $R \simeq 1390$ and a sampling of $1.6 \AA$ per pixel for a slit width of $1^{\prime \prime}$. The total slit length varied in order to allocate as many objects as possible but still allow for an accurate sky subtraction. Compared to previous observations where the length was always kept longer than 10-12" (e.g., LP11) in some cases our slits were as short as 8 ". The objects were placed at the center of the slit whenever allowed by geometrical constraints (approximately in $90 \%$ of the cases) and in all cases at least 4 " from the border.

The observation strategy was almost identical to the one adopted in LP11 and LP14: series of spectra with $1200 \mathrm{~s}$ 


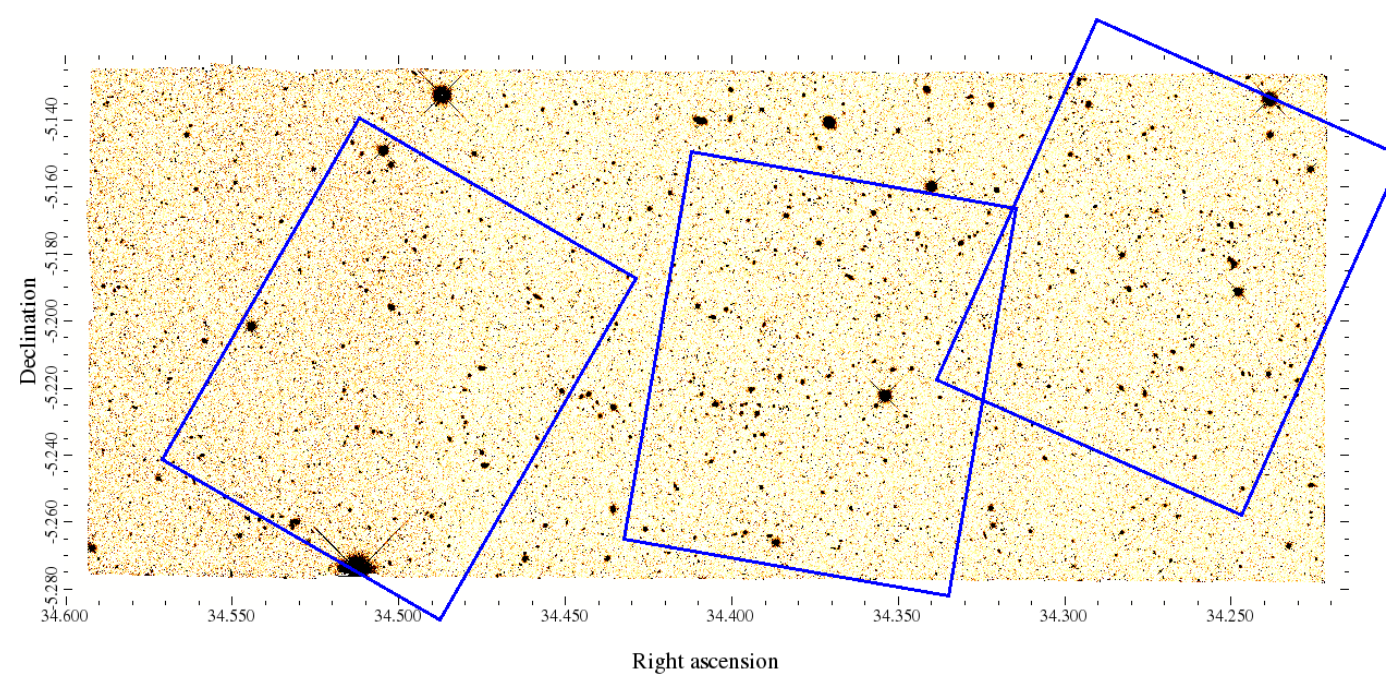

Fig. 2. Layout of the three FORS2 masks in the UDS field overplotted on the CANDELS $H_{160}$ image.

Table 1. Mask characteristics.

\begin{tabular}{ccccccccc}
\hline \hline Mask & $\begin{array}{c}\text { RA } \\
\mathrm{J} 2000\end{array}$ & $\begin{array}{c}\text { Dec } \\
\mathrm{J} 2000\end{array}$ & $\begin{array}{c}T_{\exp } \\
\mathrm{h}\end{array}$ & $z \sim 6$ & $z \sim 7$ & AGN & Others & $N_{\text {tot }}$ \\
\hline UDS1 & $2: 17: 29.6$ & $-05: 57: 22.4$ & 12.2 & 6 & 9 & 11 & 5 & 31 \\
UDS2 & $2: 17: 59.9$ & $-05: 12: 52.1$ & 12.2 & 11 & 6 & 8 & 3 & 28 \\
UDS3 & $2: 17: 04.5$ & $-05: 11: 03.3$ & 12.2 & 5 & 11 & 3 & 11 & 30 \\
COSMOS1 & $10: 00: 30.7$ & $+02: 16: 56.9$ & 12.2 & 12 & 10 & 7 & 3 & 32 \\
COSMOS2 & $10: 00: 37.4$ & $+02: 27: 10.7$ & 12.2 & 5 & 12 & 5 & 3 & 25 \\
COSMOS3 & $10: 00: 22.5$ & $+02: 23: 52.4$ & 12.2 & 10 & 8 & 5 & 5 & 28 \\
GOODS-S1 & $03: 32: 17.9$ & $-27: 45: 54.4$ & 20 & 18 & 18 & 3 & 1 & 40 \\
GOODS-S2 & $03: 32: 39.6$ & $-27: 46: 02.8$ & 20 & 12 & 15 & 2 & 2 & 31 \\
\hline TOTAL & & & & 78 & 89 & 44 & 33 & 245 \\
\hline
\end{tabular}

integration (instead of the $665 \mathrm{~s}$ that were used in previous observations) were taken at two different positions, offset by 4 " (16 pixels) in the direction perpendicular to the dispersion, in the pattern ABBA. The total net integration time was approximately $12 \mathrm{~h}$ for the masks in the UDS and COSMOS fields, and $20 \mathrm{~h}$ for the masks in the GOODS-South field (the precise values for each mask are reported in Table 1). Observations were carried out in service mode and we requested a seeing limit of $0.8^{\prime \prime}$, clear conditions, and moon illumination below 0.3 . The observations were spread over six semesters, from January 2013 to January 2016.

Data were reduced using the well-tested pipeline developed by Vanzella et al. (2008) and described at length in Vanzella et al. (2014), specifically tailored for the reduction of very faint spectra, which we already used for all our previous observations of $z \sim 7$ galaxies (V11; LP11; LP14). Briefly, after a standard flat-fielding and bias subtraction, the sky background was subtracted between consecutive exposures, exploiting the fact that the target spectrum is offset due to dithering. An alternative sky-subtraction method was also applied consisting in fitting a polynomial function to the background, giving similar or slightly worse results. A standard wavelength calibration was performed using arc lamps (He, Ar) that provide sharp emission lines over the entire spectral range observed. Before combining frames, particular care was devoted to the possible offset along the wavelength direction by measuring the centroids of the sky lines in the wavelength interval 9000-9900 A. Systematic translations of the wavelength scales were corrected. The one-dimensional (1D) spectra were extracted using apertures which encompassed all the Ly $\alpha$ flux based on individual visual inspection. Finally, the 1D spectra were flux-calibrated using observations of spectro-photometric standard stars. Based on the analysis of the standard stars observed with the same setup of science targets, we derive that the relative error due to flux calibration is less than $10 \%$.

We expect slit losses to be small, given the extremely compact size of the targets, the very good image quality during the observations, and the extremely careful centering procedure during the production of the masks. To give an estimate of the possible effect, we refer to the simulation by Lemaux et al. (2009), who showed how the slit losses depend both on image quality and on the size of the objects. We retrieved the sizes of the galaxies $r_{\mathrm{e}}$, measured in the $J_{125}$ band using Galfit (van der Wel et al. 2012). The median $r_{\mathrm{e}}$ for our detected objects is $0.148^{\prime \prime}$, in agreement with the average size of $z \sim 7$ galaxies determined by Grazian et al. (2012) also in the $J_{125}$ band. As previously specified, the upper limit for the seeing requested by the survey was $0.8^{\prime \prime}$ and in $80 \%$ of the cases our OBs were graded A, implying that all constraints were respected (with seeing between 0.4 and $0.8^{\prime \prime}$ ). Only for the remaining $20 \%$ of the OBs was the seeing between 0.8 and $1.0^{\prime \prime}$. According to the simulation of Lemaux et al. (2009; Fig. 3 of that paper), for these conditions and target sizes, slit losses should be about $15 \%$. Since all masks contained a random mixture of $z \sim 6$ and $z \sim 7$ sources, the average seeing conditions are the same for the two samples, and the 


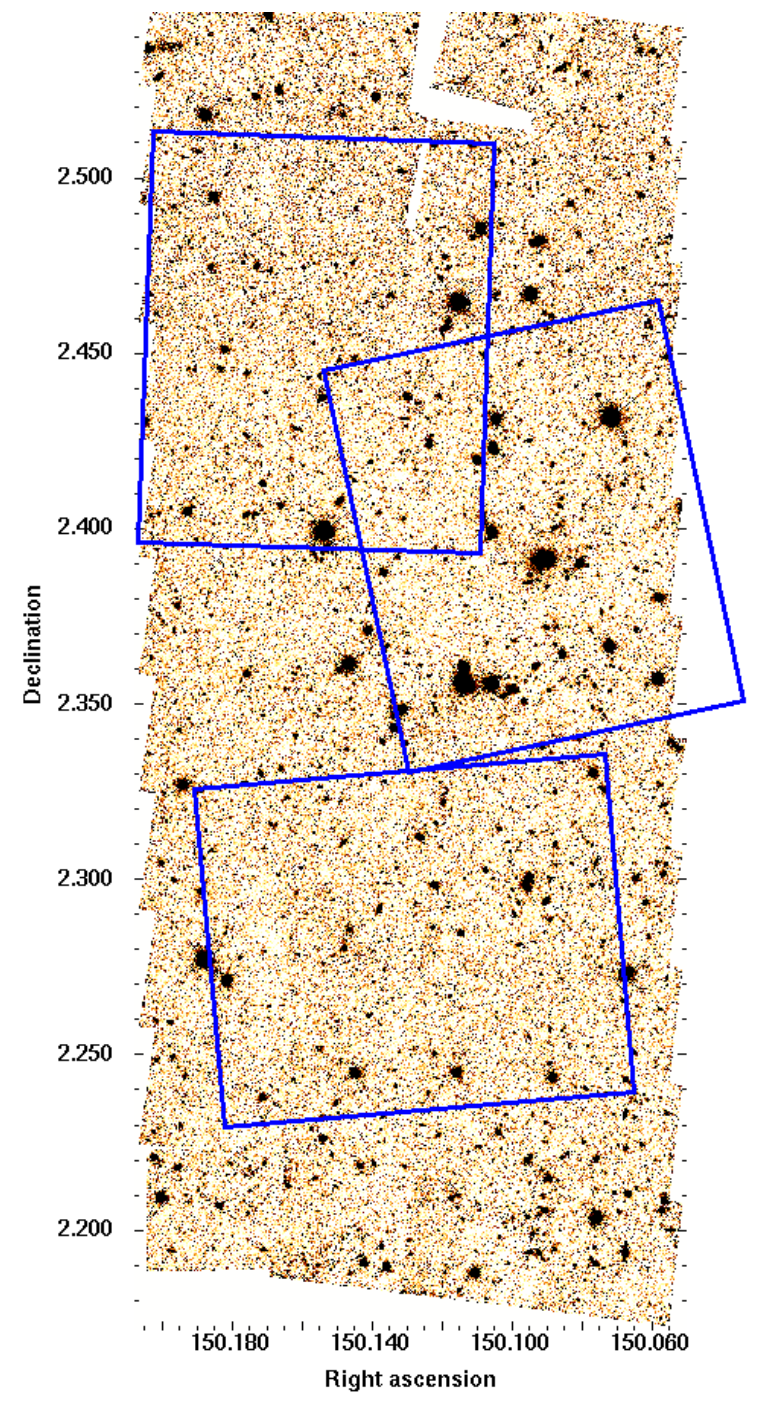

Fig. 3. Layout of the three FORS2 masks in the COSMOS field overplotted on the CANDELS $H_{160}$-band image.

sizes do not change appreciably between these two redshifts. In conclusion, we do not take into account possible slit losses in the subsequent discussion. However we are aware that this may not be true in all cases, as recently shown by the discrepancy found between the fluxes measured by HST grism and ground-based slit spectroscopy in some high-redshift galaxies (Huang et al. 2016; Tilvi et al. 2016; Hoag et al. 2017).

In some cases, the sky-subtraction was not optimal; for example, when the slit was particularly short, or there was a lower-redshift interloper falling within the same slit, or there was a very bright object just outside the slit, or finally the target was close to the border of the slit. For these reasons we were not able to detect or set significant limits for about $7-10 \%$ of the objects observed.

In this paper we also report new results from archival observations that were taken as part of the program ESO 088. A-1013 (PI Bunker). This program employed the same observational setup used by our large program, with a total net integration time of $27 \mathrm{~h}$ on a single mask. It observed a mixture of $i$ - and $z$-dropouts in the Hubble Ultra Deep field. The description of this program and some of the results were presented by Caruana et al. (2014). We applied the same color criteria described above to their targets (the target list was derived using the information contained in the headers), and re-reduced the data in the relevant slits with our own pipeline, which is particularly tailored to the detection of faint emission lines. We were able to confirm several additional high-redshift sources, which were also in our selection catalogs, with the most distant one already reported in our previous work (CANDELS 34271 at $z=6.65 ;$ LP14).

\section{Results}

We searched for spectral features by an automatic scanning of the two-dimensional (2D) spectra complemented by visual inspections by LP and EV. We only report features that were confirmed independently by LP and EV. Before validating a detection, we also ensured: 1) that the position of the putative line in the $2 \mathrm{D}$ frame was fully consistent with the position of the target along the slit in the mask; 2) that in none of the individual 2D frames were there spikes or artifacts at the position of the line; 3 ) the presence of negative residuals at positions \pm 16 pixels along the $Y$-axis in the 2D frame for features with $S / N \geq 5$, as a further confirmation of the reality of the feature. In the spectra of 56 objects we detect a single emission line that we interpret as $\operatorname{Ly} \alpha$ (see the detailed discussion below). In one further case (COSMOS ID 8474) we detect two emission lines that are consistent with $\mathrm{H} \beta$ and [OIII] from a lower-redshift galaxy at $z=0.661$. This object had been selected both by the $i$-dropout criteria and for its photometric redshift. In one further galaxy, GOODS-S ID 31759 we detect two emission lines that are separated by $6 \AA$ and are consistent with the [OII] emission doublet $(3726 / 3729 \AA)$ from a galaxy at $z=1.393$. This would be a low-luminosity [OII] emitter with $\log (\mathrm{L}([\mathrm{OII}]))=41.2$. However there is a strong sky-line equidistant between the two components: therefore it could also be possible that the line is actually $\operatorname{Ly} \alpha$ at $z=6.34$, which appears split into two components because of the strong sky-line. In this latter case the line would be intrinsically very bright since the measured flux (with no correction) is already $1.7 \times 10^{-17} \mathrm{erg} \mathrm{s}^{-1} \mathrm{~cm}^{-2}$. The broad-band photometry of this object actually strongly favors the high-redshift solution, with the photometric redshift constrained between 6.02 and 6.33 at $68 \%$ confidence. In Fig. 5 we show the broad-band photometry of the galaxy together with the bestfitting SED, at $z=1.393$ and $z=6.34$, respectively: the high redshift solution better matches the photometry, especially if we include the contribution from nebular emission (blue line).

In all other cases, for single emission line spectra, alternative identifications have been discarded with good confidence for the following reasons: 1) The wavelength range covered by our spectra is large enough that if the detected lines were $\mathrm{H} \alpha$, $\mathrm{H} \beta$, or either of the two [OIII] 5007,4959 $\AA$ components, there would be at least another line visible in the same spectrum, unless the object had very anomalous line ratios. 2) A possible identification with [OII], which could be naturally consistent in some targets with photometric redshift around 1.2-1.3, is also not likely since we would resolve the doublet at 3727-3729 $\AA$, as is the case for the target described above. In Vanzella et al. (2011) we showed other examples of such low-redshift doublets clearly resolved in the observations (Fig. 2 of that paper). We note that this is only partially true in a few cases where the detected line falls very close to a sky-line and the second component of the doublet could fall on top of the sky-line. 3) When the $\mathrm{S} / \mathrm{N}$ is high enough (17 targets), a sharp asymmetry of the line is observed. This shape is typical only of Ly $\alpha$ at high redshift and it is not observed for any other emission line. 4) For 

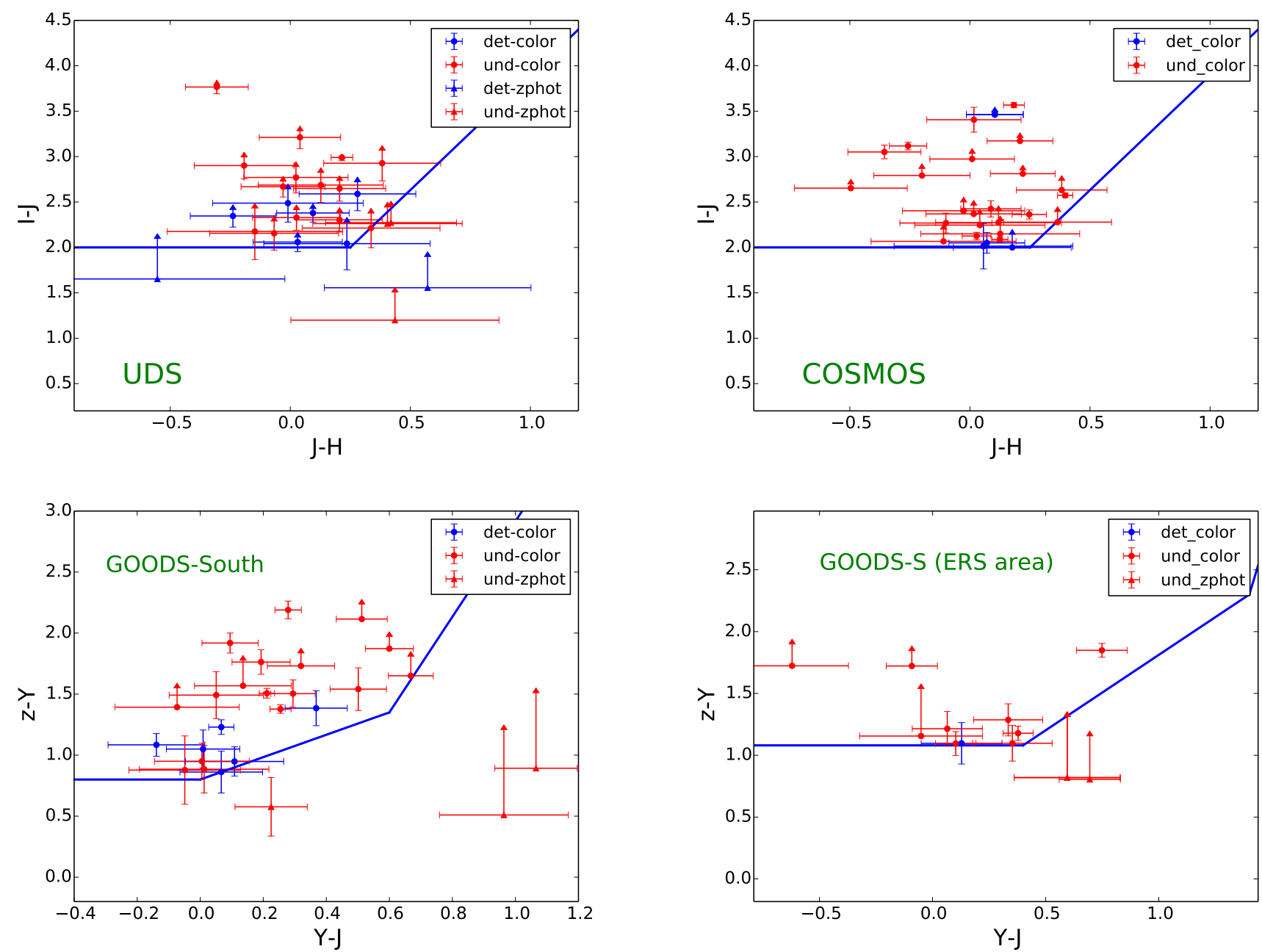

Fig. 4. Color-color selection diagrams in the different regions observed in this work. Upper panels: $J_{125}-H_{160}$ vs. $I-J_{125}$ diagram for the UDS field (left) and the COSMOS field (right). Bottom panels: $Z_{850}-Y_{105}$ vs. $Y_{105}-J_{125}$ diagram for the GOODS-South field (left) and the $Z_{850}-Y_{095}$ vs. $Y_{095}-J_{125}$ for the ERS area (right). The blue circles are the detected objects, and the red circles are the undetected ones. Blue and red triangles represent, respectively, confirmed and unconfirmed objects that were selected only by means of their photometric redshift. The solid lines are the color cuts representing the different criteria described in Sect. 2.

the brightest objects (approximately $H \leq 25.5$ ) we also observe a weak continuum redward of the line but not blueward of it, which would not be observed for lower-redshift objects and is perfectly consistent with a large drop observed between the $z$ and $Y$ (or $i$ and $z$ ) broad-band photometry. The redshifts are determined from a fit of the $\operatorname{Ly} \alpha$ line peak. The errors, estimated following Lenz \& Ayres (1992) depend on the resolution and on the $\mathrm{S} / \mathrm{N}$ of the line and are typically $\sim 0.002$ for the faintest line emitters $(S / N \sim 5)$ and $<0.001$ for the brightest sources $(S / N>10)$.

In the spectra of nine further galaxies, typically our brightest candidates around $z \sim 6$, we detect only weak continuum with a sharp break but no emission line. We interpret this discontinuity as the Ly $\alpha$ forest break. To estimate the wavelength of the break we smooth both the 1D and 2D spectra and we determine the wavelength at which the flux becomes consistent with zero (i.e., the noise) by searching for a change in slope in the cumulative sum of the flux (e.g., Watson et al. 2015). These redshifts are obviously less accurate then those based on the Ly $\alpha$ emission line. The uncertainty is derived by changing the smoothing parameter and repeating the above measurement and it is \pm 0.1 . In these cases, we have further confidence that the redshift assignment is correct given the good agreement between the photometric redshift and the spectroscopic one, hence excluding with high probability that the break in the continuum is tracing the $4000 \AA$ break, potentially associated with a lower-redshift solution.

Taking into account the objects which had problems in the data-reduction process (as detailed in Sect. 3), the overall success rate for redshift determination of the Large Program for high-redshift targets was $\sim 40 \%$. In Figs. $11-13$ we show the 2D spectra of all the confirmed galaxies.

We assigned a quality flag (QF) to each spectrum, indicating the reliability of the redshift identification which is mainly due to the S/N of the Ly $\alpha$ line: "A" indicates a completely secure identification (when we observe a clear asymmetry of the line and in some cases the continuum red-ward of it) and "C" indicates the most uncertain, typically assigned to those objects where we only observe a weak continuum and a break. A similar redshift flag scheme was adopted previously by Vanzella et al. (2008) for the GOODS south spectroscopic campaign. The flags were first independently assigned by LP and EV and then an agreement was reached in case of initial discrepancies.

In Tables 3-5 we report the redshift identifications for a total of 67 objects, 21, 18, and 28, in the UDS, COSMOS, and GOODS-South fields, respectively. These are the 


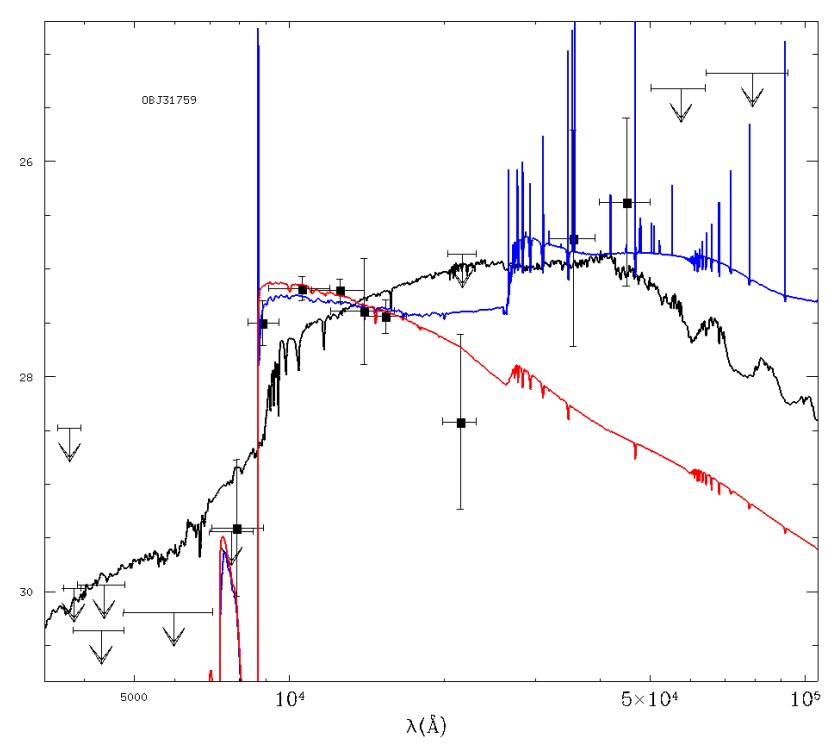

Fig. 5. Observed photometry of object GOODS-S 31759 with the best fit solutions at the two possible spectroscopic redshifts of $z=1.393$ (black curve) and $z=6.34$ (red curve and blue curve) corresponding respectively to the identification of the emission line visible in the spectrum as the [OII] doublet or the Ly $\alpha$ line. The two SEDs for the high-redshift solution correspond to models with and without nebular contribution (blue and red, respectively).

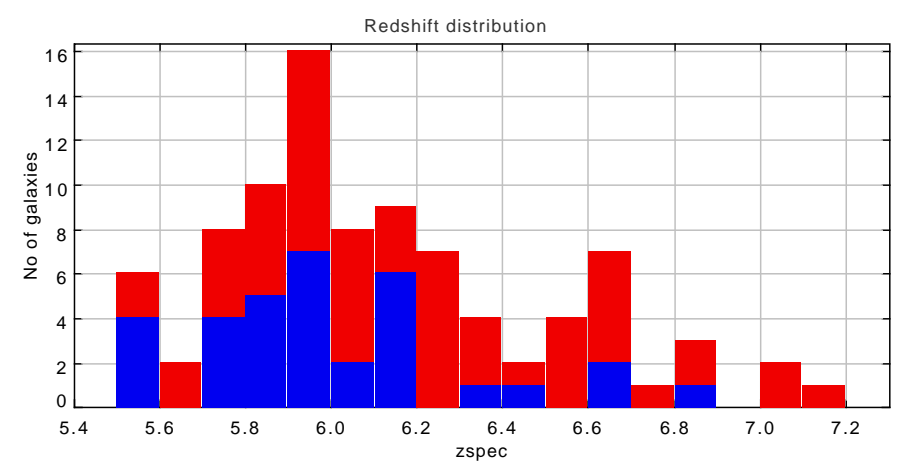

Fig. 6. Spectroscopic redshift distribution for all confirmed galaxies in the three CANDELS fields analyzed in this work between $z=5.5$ and 7.2. In blue we show previous redshifts in these fields from the literature (Pentericci et al. 2011, 2014; Caruana et al. 2014; Curtis-Lake et al. 2012; Fontana et al. 2010; Vanzella et al. 2008), while in red we show the new redshifts from this work.

confirmed galaxies at $z \sim 6$ and 7 plus the two lower-redshift interlopers discussed above; we will report on AGNs and other filler galaxies in future works. For each object, we report the CANDELS ID, the RA and Dec, the CANDELS $H_{160^{-}}$ band magnitude, the official CANDELS photometric redshift (Santini et al. 2015 for the GOODS-South and UDS fields, Nayyeri et al. 2017 for the COSMOS field), the effective radius $r_{\mathrm{e}}$ in the $J_{125}$ band (van der Wel et al. 2012), and the spectroscopic redshift with its quality flag. For the objects with an identified Ly $\alpha$ emission line, we report the total flux measured from the 1D spectra, and the Ly $\alpha$ rest-frame EW (REW). This last quantity is determined by estimating the continuum emission at $1300 \times(1+z) \AA$ from the available broad-band photometry, extrapolating from the nearest filter using a power law with the appropriate $\beta$ slope (see below). We remark that we do not apply any slit loss correction to the Ly $\alpha$ flux: our objects are mostly very compact and the slit losses should be minimal, however the reported REW might be in some cases underestimated, particularly for the few objects with large sizes. The fluxes of the Ly $\alpha$ lines vary between $1.5 \times 10^{-18}$ and $2 \times 10^{-17} \mathrm{erg} \mathrm{s}^{-1} \mathrm{~cm}^{-2}$ and the REW span a range from 3 to $110 \AA$. For the objects with no Ly $\alpha$ emission, we report a limit $(3 \sigma)$ on the REW that is derived using accurate simulations of the reduction process presented in Pentericci et al. (2014) and Vanzella et al. (2014) and assuming that the undetected $i$-dropouts are exactly at $z=6$ and the undetected $z$-dropouts are at $z=6.9$.

In the tables we also list the absolute UV magnitude, $M_{\mathrm{UV}}$, and the slope $\beta$ of the UV continuum. These parameters are obtained by the common power-law approximation for the UV spectral range $F_{\lambda} \propto \lambda^{\beta}$, and estimated by fitting a linear relation through the observed $\mathrm{AB}$ magnitudes of the objects, excluding the band that contains the Ly $\alpha$ emission line, that is, the $z$-band for objects around $z \sim 6$ and the $Y$-band for higher-redshift objects. We use broad-band fluxes measured in $2 \times$ FWHM apertures instead of the isophotal photometry to estimate the UV slope and $M_{\mathrm{UV}}$, after verifying that this choice improves the stability of the $\log (F)$ versus $\log (\lambda)$ fit (Castellano et al. 2012), compared to simply using the CANDELS isophotal magnitudes. Finally in Tables 3-5 we also indicate the selection criteria for each target (second-to-last column): " 1 " is the $z$-dropout color, " 2 " is the $i$-dropout color, and " 3 " is the photometric redshift. The distribution of the new redshift identifications is presented in Fig. 6, together with previously known spectroscopic redshift in the three CANDELS fields from previous works (Caruana et al. 2014; Pentericci et al. 2011，2014; Curtis-Lake et al. 2012; Fontana et al. 2010; Vanzella et al. 2008) in the same redshift range.

The data on the high-redshift objects will be released through the ESO science archive facility. We plan to release both the 2D spectra presented in Figs. 11-13 as well as the 1D extracted spectra, with associated noise spectra.

\section{Properties of confirmed galaxies}

\subsection{Accuracy of photometric redshifts}

We wish to quantify the percentage of outliers and the accuracy of the CANDELS photometric redshift, that is, the mean offset between $z_{\text {spec }}$ and $z_{\text {phot }}$ (bias) and the rms based on our spectroscopic samples. To enlarge our statistics, we include additional CANDELS galaxies in our three fields with published spectroscopic redshift at $z \geq 5.5$ from Pentericci et al. (2011, 2014) and Caruana et al. (2014), mostly located in the GOODSSouth field. We have also checked the very recent results from the MUSE Wide field survey (Herenz et al. 2017; Caruana et al. 2018): from the 11 published spectroscopic redshifts in the range $5.5<z<6$, only 4 are detected in the CANDELS catalog, with the others being below the CANDELS detection limit; of these, one is a new identification (ID $7538 z=5.52027$ ). Finally we checked the DR1 of the VUDS survey (Le Fèvre et al. 2015): of the 6 galaxies with spectroscopic redshift $>5.5$ and good quality flag ( $\mathrm{QF} \geq 2), 3$ are newly identified galaxies in the COSMOS field.

In Fig. 7 (left panel) we plot the spectroscopic redshift of all these galaxies and compare them to the photometric redshifts obtained by the CANDELS team. The targets are color-coded depending on the field. The error bars in the photometric redshift represent the $68 \%$ upper and lower uncertainties. It is evident that there is a small fraction of objects with low photometric 

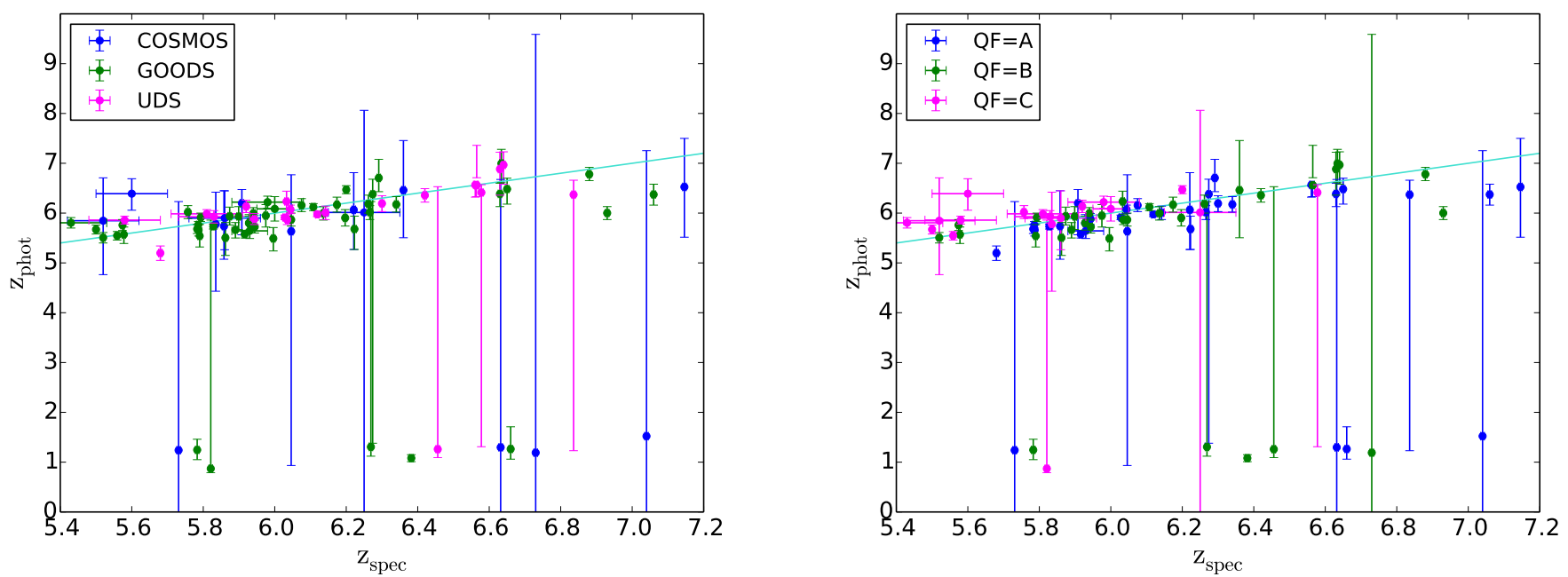

Fig. 7. Spectroscopic redshift vs. CANDELS photometric redshifts for the objects presented in this paper, left panel: represented with different color codes for the three different fields (COSMOS, GOODS-South and UDS in blue, green and red respectively), right panel: with different color codes for the different quality flags (flag A, B and C as blue, green, red respectively). The error bars represent the $68 \%$ upper and lower uncertainties of the photo- $z$ (see Dahlen et al. 2013 for details).

redshift, typically around 1 , but high spectroscopic redshift: these objects were selected from the color criteria. For most of these objects the photometric redshift uncertainty at $68 \%$ is very large and includes the high-redshift solution compatible with the real spectroscopic redshift. The relative number of discrepant objects is higher for the COSMOS field (blue symbols) where the photometric redshifts are indeed somewhat less accurate because of the fewer deep photometric bands available. In the right panel of the figure, we show the same plot but we colorcode the targets depending on the QF of the spectroscopic redshift. We notice that most of the galaxies with the lowest $\mathrm{QF}$ are at $z<6.4$. These are typically the objects where the redshifts are determined from the Ly $\alpha$ break.

In Fig. 8 we plot the $\Delta z=\left(z_{\text {spec }}-z_{\text {phot }}\right) /\left(1+z_{\text {spec }}\right)$ versus the $H_{160}$-band magnitude (upper panel) and versus $z_{\text {spec }}$ (lower panel). If we define the catastrophic outliers as the objects for which $\Delta z>0.15$ (e.g., as in Dahlen et al. 2013), the fraction of such galaxies is $14 \%$, which is substantially higher than what is found at lower redshift for the rest of the CANDELS cat$\operatorname{alog}(\sim 3 \%$, see Dahlen et al. 2013). The fraction of outliers is higher at fainter magnitudes and higher redshifts. After removing the outliers, the bias, that is, the mean of $\Delta z$, is 0.007 . The bias is not constant but depends on the redshift and magnitudes of the sources. In particular, for the most distant objects $(z>6.8)$ and for galaxies fainter than $H \sim 27$, we always find $z_{\text {spec }} \geq z_{\text {phot }}$, with a mean bias of 0.07 and 0.04 , respectively. The bias cannot be produced by the uncertainties in the spectroscopic redshift measurements, since all the objects with the largest uncertainty are actually the brightest sources in the lowest redshift range. Since objects at $z>6.8$ are strong line emitters, one possible explanation is that the presence of the line influences the determination of photometric redshifts. Similar offsets were found, for example, by Oyarzún et al. (2016) for LAEs at $z \sim 4$ and by Brinchmann et al. (2017) for galaxies with $z>3$ and $H<27$ in the MUSE deep field. After removing the catastrophic outliers, the $\sigma$ of the distribution is 0.036 . Therefore while the outliers fraction is about three times higher than that of the entire CANDELS catalog at all redshifts, the rms is not much higher even for the most distant sources.
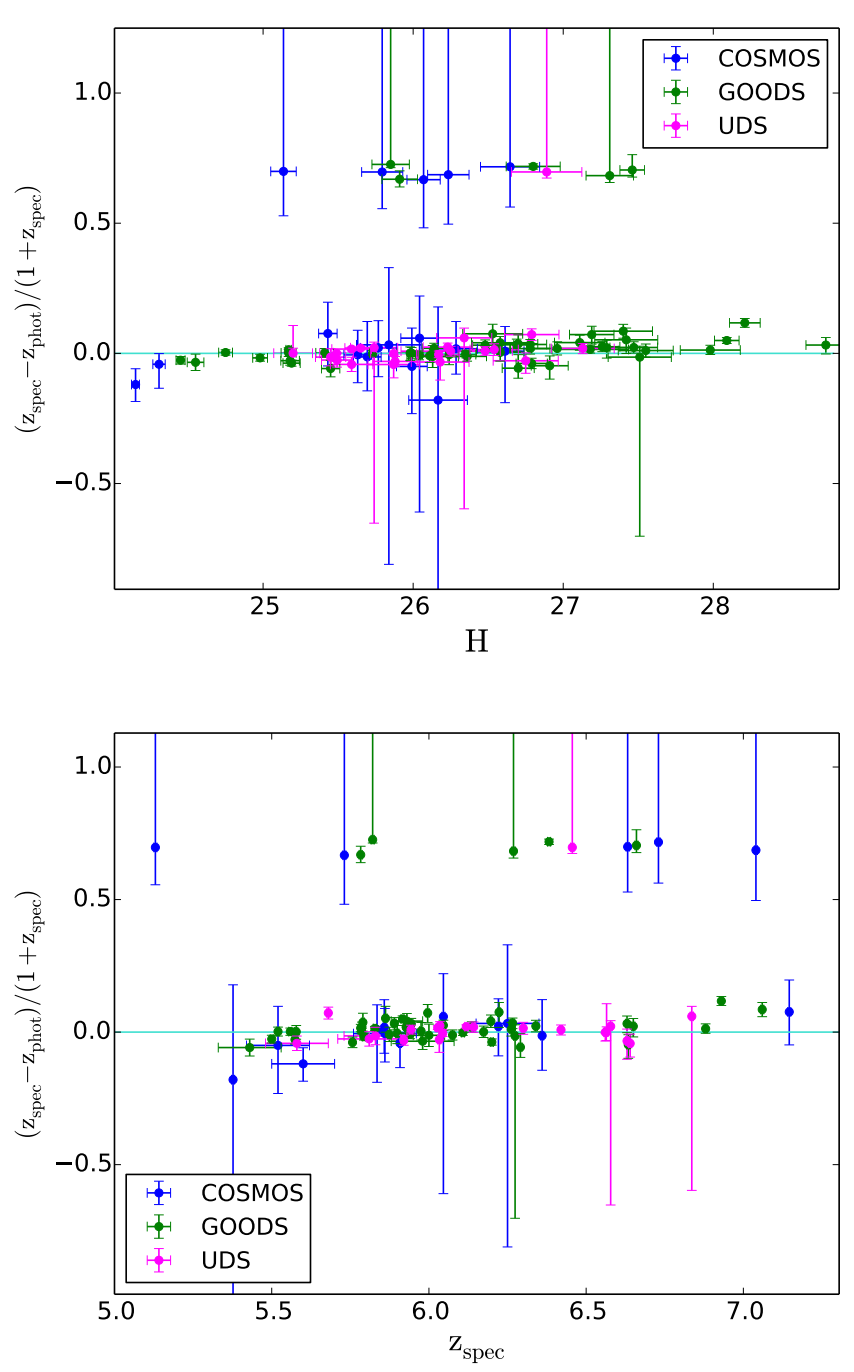

Fig. 8. $\Delta z=\left(z_{\text {spec }}-z_{\text {phot }}\right) /\left(1+z_{\text {spec }}\right)$ as a function of total $H$-band magnitude (top panel) and $z_{\text {spec }}$ (bottom panel). The blue symbols are galaxies in the COSMOS field, the green ones are in the GOODS-South field and the pink ones in the UDS field. 


\subsection{Ly $\alpha$ rest-frame $E W$ distribution at $z=6$ and 7}

Using the new CANDELSz7 observations and previous samples, we can now derive the Ly $\alpha$ REW distributions both at $z \sim 6$ and $z \sim 7$ as well as the fractions of Ly $\alpha$ emitting LBGs for faint and bright galaxies. Since we want to obtain results that are as robust as possible and are not subject to, for example, field to field variations, we enlarge our statistics by including all previous observations available, both from our group and from the literature. Clearly this means that the resulting sample is less homogeneous than CANDELSz7 alone, but this is compensated by the higher number of galaxies observed, especially at faint magnitudes with the inclusion of lensed objects. In addition, the larger number of independent fields included mitigates uncertainties due to field-to-field variations that are very important in a partially neutral Universe (e.g., Jensen et al. 2013). In the following, we first describe in more detail the sample at $z=6$ and $z=7$ that we use. We then discuss the derivation of the REW limits for all galaxies, with the help of dedicated simulations, and finally we derive the REW distributions and Ly $\alpha$ emitters fractions in the two redshift bins.

\subsubsection{The samples at $z=6$ and $z=7$}

At $z=6$ we use the sample that is described extensively in De Barros et al. (2017): briefly, it consists of 127 galaxies selected as $i$-dropouts, of which $79(>62 \%)$ have a confirmed redshift between 5.5 and 6.5, mostly from the $\operatorname{Ly} \alpha$ line and in a few cases from the Lyman break. The galaxies come from our new program CANDELSz7 as well as previous works mostly by our team (Pentericci et al. 2011, 2014; Caruana et al. 2014; Vanzella et al. 2008, 2011; Fontana et al. 2010). Most galaxies have been selected from the CANDELS fields (GOODS-South, UDS and COSMOS), with a small subset coming from the NTT and BDF fields (Castellano et al. 2010b). All galaxies have been observed with FORS2, although the integration times are different and the set up of the earlier observations by Vanzella et al. (2008) had a lower resolution.

At $z=7$ we started from the sample that we previously assembled and analyzed in LP14 and add galaxies observed within CANDELSz7 and selected with the color criteria described in Sect. 2. This sample consists of 134 objects, of which $30(22 \%)$ have a confirmed redshift between 6.5 and 7.2, all from the Ly $\alpha$ emission line. In this sample, galaxies come from nine independent fields, namely UDS, GOODSSouth, COSMOS, BDF and NTT (see previous references), with the addition of a few objects from the Subaru XMM Deep Survey (SXDF) and GOODS-North from Ono et al. (2012), ten lensed galaxies from the observations of the Bullet cluster (Bradač et al. 2012), and some lensed galaxies from the Abel1703 field (Schenker et al. 2012). All data, with the exception of those by Ono et al. (2012) and Schenker et al. (2012), were obtained using FORS2 with the same setup employed for CANDELSz7, and variable integration times ranging from a minimum of 10 to a maximum of $27 \mathrm{~h}$.

\subsubsection{The fraction of Ly $\alpha$ emitters in LBGs}

To accurately derive the fractions of $\operatorname{Ly} \alpha$ emitters in our sample, we must first estimate the REW limits for each target (both detected and undetected). To do this we employ the simulations which were extensively discussed in Vanzella et al. (2014) and Pentericci et al. (2014) and were specifically tailored for observations with the FORS2 $600 \mathrm{z}$ grism (see in particular Fig. 3
Table 2. Fractions of $\operatorname{Ly} \alpha$ emitters at $z=7$.

\begin{tabular}{lccc}
\hline \hline Mag range & REW $>25 \AA$ & REW $>50 \AA$ & REW $>75 \AA$ \\
\hline$-21.25<M_{\mathrm{UV}}<-20.25$ & $0.09_{-0.04}^{+0.07}$ & $0.07_{-0.03}^{+0.05}$ & $<0.04$ \\
$-20.25<M_{\mathrm{UV}}<-18.75$ & $0.14_{-0.07}^{+0.11}$ & $0.10_{-0.05}^{+0.09}$ & $0.06_{-0.04}^{0.08}$ \\
All & $0.10_{-0.03}^{+0.05}$ & $0.06_{-0.02}^{+0.03}$ & $0.02_{-0.01}^{+0.02}$ \\
\hline
\end{tabular}

of Pentericci et al. 2014) and take into account the instrument throughput and the presence and strength of the skylines. The limit achieved on the $\operatorname{Ly} \alpha$ line depends on the integration time, on the continuum flux of the objects, and also on the exact redshift of the line. For the objects without a confirmed redshift we assume $z=6$ for the $i$-dropouts and $z=6.9$ (the approximate mean of the redshift PDF) for the $z$-dropouts. For the objects with a detected Ly $\alpha$ emission line or with a Lyman break, we also determine the $3 \sigma$ limit of the line at the exact redshift position. For the Ono et al. (2012) sample, we use the $3 \sigma$ limits on the flux reported in Table 2 of their paper, while for the four objects by Schenker et al. (2012) we derive approximate $3 \sigma$ limits from the information provided in the paper. To convert the $3 \sigma$ limit on the emission line flux to REW limits, we used the HST photometry to determine the continuum flux.

In calculating the Ly $\alpha$ fractions, we must also consider that for some galaxies the redshift probability distribution extends well beyond $z \sim 7.3$, which is the limit out to which we can detect the $\operatorname{Ly} \alpha$ emission in the FORS 2 observations. In particular, the ten Bullet cluster candidates observed by Bradač et al. (2012) were selected in such a way that the probability of galaxies being at $z>7.3$ is quite high, $\sim 48 \%$ (see Fig. 5 in Hall et al. $2012)$. This is due to the broad $J$-band filter $\left(J_{110}\right)$ that was available for the selection. Therefore we weighted each object in the $z \sim 7$ sample by evaluating the total probability of the galaxy being outside the redshift range that is observable by the spectroscopic setup. In practice, for most of the sub-samples this probability is negligible (see Fig. 6 in Ouchi et al. 2010 for the Ono et al. sample, and Fig. 7 in Castellano et al. 2010a for the NTT, GOODS-South, and BDF samples), while it is $\simeq 16 \%$ for the UDS and COSMOS samples (which have a tail to $z \simeq 8$; see Fig. 1 in Grazian et al. 2012) and 48\% for the Bullet cluster sample. No weight was applied to the $z \sim 6$ sample since the redshift distribution of these targets is entirely contained in the FORS2 observed range.

To derive the fraction of galaxies with Ly $\alpha$ emission at various REW limits, we proceed as follows: for each REW value we consider only those objects that have observations deep enough to probe this limit, given their magnitude and redshift, and regardless of whether they have a detected line or not. This means that if an object has a detected Ly $\alpha$ with REW $=50 \AA$ but its observations were only deep enough to probe REW > $30 \AA$, this galaxy is not considered when evaluating the fractions with lower REW limits. The resulting fractions of galaxies with Ly $\alpha$ REW > $25 \AA, 50 \AA$ and $75 \AA$ are presented in Table 2, for faint $\left(-20.25<M_{\mathrm{UV}}<-18.75\right)$ and bright $\left(-21.25<M_{\mathrm{UV}}<\right.$ -20.25) galaxies separately, using the same magnitude bins as in LP14, and for all galaxies in the sample. The errors were evaluated using the statistics for small numbers of events by Gehrels (1986).

Thanks to our very large samples, not only can we determine the fractions of emitters above these commonly used thresholds ( $25 \AA, 50 \AA$ and $75 \AA$ ), but we can also derive accurate cumulative distributions which are presented in Fig. 9 for all galaxies (left panel) and for galaxies fainter than $M_{\mathrm{UV}}=$ 

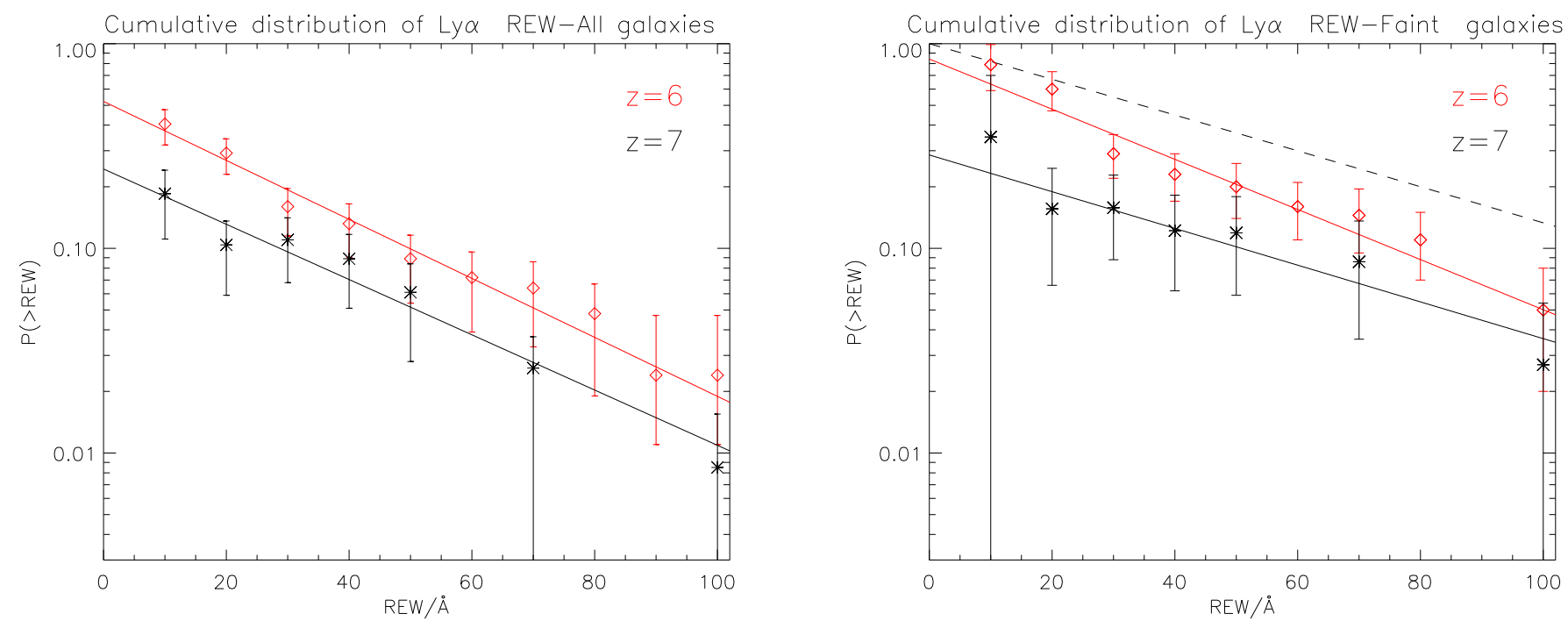

Fig. 9. Left panel: cumulative distribution of Ly $\alpha$ REW $P(>$ REW) for the complete sample of redshift 6 and 7 galaxies in red and black, respectively. The lines are the best fit exponential functions of the two distributions. Right panel: same distribution but only for galaxies with $M_{\mathrm{UV}}>-20.25$ (the faint sample). The dashed line indicates the previously adopted fit to the distribution at $z \sim 6$ (e.g., Dijkstra et al. 2011; LP14).

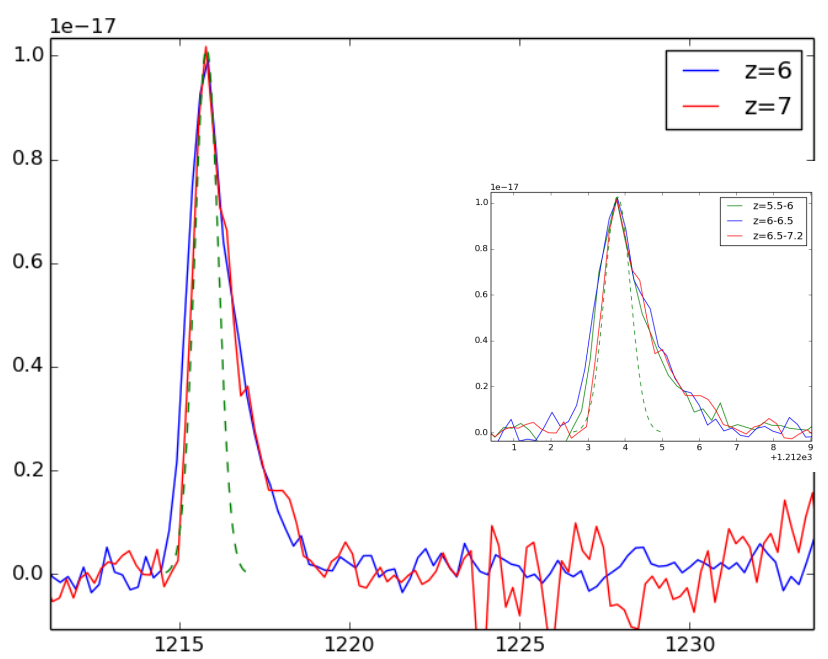

Fig. 10. Stacks of the $z \sim 6$ and $z \sim 7$ sources (in blue and red respectively) showing a very asymmetric $\operatorname{Ly} \alpha$ line in both cases: the $z \sim 6$ stacked line is broader than the higher-redshift line. The dashed green line is the resolution of the FORS2 spectra. We also note the positive continuum redward of the line for the $z \sim 6$ stack, while in the $z \sim 7$ case the flux is consistent with the noise. In the inset, the Ly $\alpha$ line from the stacks, with the lower-redshift sample split into two bins (5.5-6 in blue and 6-6.5 in green), which are consistent with each other.

-20.25 (right panel). The distribution of $\operatorname{Ly} \alpha$ emission at lower redshift is usually represented with an exponential function $P(>\mathrm{REW}) \propto \exp \left[-\mathrm{REW} / \mathrm{REW}_{0}\right.$ ] (e.g., Gronwall et al. 2007; Guaita et al. 2010). In the same figures we also plot the best fit exponentials that match the observations: these have scales $\mathrm{REW}_{0}=32 \pm 8$ and REW $033 \pm 7$ at redshifts 6 and 7, respectively. For the faint galaxies, we obtain $\mathrm{REW}_{0}=35 \pm 10$ and $\mathrm{REW}_{0}=48 \pm 22$ at redshifts 6 and 7, respectively.

\subsubsection{Comparison to previous results}

At $z \sim 7$ we can compare the fractions of line emitters from the new sample to our previous derivation in LP14: the new fractions are slightly lower than the previous values for REW $>25 \AA$ and very similar, within the uncertainties, for the higher REW limits, both for faint and bright galaxies (see Table 2 of LP14).

At $z \sim 6$, and as already extensively discussed in De Barros et al. (2017), the fraction of Ly $\alpha$ emitters that we find are considerably below previous estimates. Specifically, previous fractions evaluated at $z \sim 6$ for galaxies with $M_{\mathrm{UV}}>-20.25$ were $0.54 \pm 0.11$ and $0.27 \pm 0.08$, for $\mathrm{EW}>25 \AA$ and $\mathrm{EW}>$ $55 \AA$ (Stark et al. 2011), respectively, while we find $0.40 \pm 0.08$ and $0.16 \pm 0.05$. This discrepancy can also be seen in Fig. 9 (left), where we plot as a dashed line the representation that was employed by Dijkstra et al. (2011) and that matched the previous fraction of $z \sim 6$ Ly $\alpha$ emitters. Our new $z \sim 6$ derivation (red line) falls below the previous one for all values above REW > $20 \AA$.

We believe that the primary explanation for the discrepancy of our $z \sim 6$ results with the previous derivations is that the selection of our sample is not biased by the presence of the emission line in the detection band. Typically, samples of high-redshift galaxies in the pre-CANDELS epoch including $i$-dropouts were selected in the $z$-band (e.g., Stark et al. 2010; Vanzella et al. 2008), which at $z \sim 6$ contains Ly $\alpha$. Therefore this positively biased the fraction of strong emitters at $z \sim 6$. Indeed our derivation of the Ly $\alpha$ distribution at this redshift, based on an $H_{160}$-band-selected sample, is lower for objects with high REW, while it is consistent for galaxies with modest Ly $\alpha$ REW (see also De Barros et al. 2017, for a more detailed discussion). Since we have not applied any slit loss correction to our Ly $\alpha$ fluxes, this could bias our Ly $\alpha$ fluxes and the REW measurements to be somewhat lower than those measured in previous works. However we do not find any clear indications of slit loss corrections applied in previous works (Schenker et al. 2012; Vanzella et al. 2009), and we highlight that the seeing conditions of our survey are excellent, given our very strict seeing limit, so we are confident that this is probably not the main reason for the discrepant results.

The fraction of Ly $\alpha$ emitters at $z \sim 6$ that we derive with the new data is similar or even slightly lower than the fraction previously found at $z \sim 5$, which was $\sim 0.48$ for the faint galaxies with REW> $25 \AA$ (Stark et al. 2011; see also Fig. 5 of De Barros et al. 2017). We will discuss the redshift evolution of the Ly $\alpha$ fraction more extensively in a follow-up paper. Here we just remark that 
Table 3. Properties of confirmed galaxies in the CANDELS UDS field.

\begin{tabular}{|c|c|c|c|c|c|c|c|c|c|c|c|c|c|}
\hline ID & $\begin{array}{c}\text { RA } \\
\text { J2000 }\end{array}$ & $\begin{array}{c}\text { Dec } \\
\text { J2000 }\end{array}$ & $H_{160}$ & zphot & zspec & $\begin{array}{c}\text { Flux } \\
\mathrm{erg}^{-1} \mathrm{~s}^{-1} \mathrm{~cm}^{2} \\
\end{array}$ & QF & $\begin{array}{c}\mathrm{EW}_{\mathrm{Ly} \alpha} \\
\AA\end{array}$ & $\beta$ & $M_{\mathrm{UV}}$ & $\begin{array}{l}r_{\mathrm{e}} \\
\prime \prime\end{array}$ & Sel & Comm. \\
\hline 1920 & 34.4887581 & -5.2656999 & 25.20 & 6.560 & 6.565 & $3.3 e-18$ & B & 3 & -2.95 & -21.93 & 0.12 & 1,3 & $\operatorname{Ly} \alpha$ \\
\hline 4812 & 34.4757347 & -5.2484999 & 25.87 & 6.566 & 6.561 & $1.9 \mathrm{e}-17$ & A & 44 & -2.37 & -20.99 & 0.15 & 1,3 & $\operatorname{Ly} \alpha$ \\
\hline 4872 & 34.4820328 & -5.2481742 & 25.49 & 6.563 & 6.564 & $5.1 \mathrm{e}-18$ & $\mathrm{~A} / \mathrm{B}$ & 10 & -1.76 & -21.30 & 0.10 & 1,3 & $\operatorname{Ly} \alpha$ \\
\hline 12123 & 34.4674568 & -5.2081060 & 25.03 & 1.261 & 5.903 & $6.5 e-18$ & A & & -1.09 & -21.35 & 0.14 & 2 & $\operatorname{Ly} \alpha$ \\
\hline 14549 & 34.4828377 & -5.1953101 & 26.75 & 6.233 & 6.033 & $5.1 \mathrm{e}-18$ & B & 20 & -2.19 & -20.12 & 0.04 & 2,3 & $\operatorname{Ly} \alpha$ \\
\hline 14715 & 34.4671059 & -5.1944642 & 25.74 & 6.413 & 6.578 & $1.3 \mathrm{e}-18$ & $\mathrm{C}$ & 4 & -1.21 & -20.87 & 0.25 & 1,3 & $\operatorname{Ly} \alpha$ \\
\hline 14846 & 34.5037651 & -5.1938372 & 25.59 & 5.912 & 6.028 & $4.8 \mathrm{e}-17$ & A & 11 & -0.93 & -20.62 & 0.09 & 2,3 & $\operatorname{Ly} \alpha$ \\
\hline 14990 & 34.3546486 & -5.1930451 & 26.54 & 6.193 & 6.297 & $7.7 e-18$ & $\mathrm{~A} / \mathrm{B}$ & 12 & -2.84 & -20.49 & 0.19 & 2,3 & $\operatorname{Ly} \alpha$ \\
\hline 15559 & 34.2315788 & -5.1897931 & 26.17 & 6.073 & 6.044 & $7.9 \mathrm{e}-18$ & A & 79 & -1.98 & -21.01 & 0.19 & 2,3 & $\operatorname{Ly} \alpha$ \\
\hline $16291^{a}$ & 34.3561440 & -5.1856260 & 25.87 & 6.967 & 6.638 & $1.5 \mathrm{e}-18$ & $\mathrm{~B} / \mathrm{C}$ & 6 & -2.47 & -20.98 & 0.18 & 3 & $\operatorname{Ly} \alpha$ \\
\hline 18087 & 34.3972206 & -5.1756892 & 25.65 & 5.974 & 6.119 & $3.2 \mathrm{e}-17$ & A & 47 & -2.14 & -21.22 & 0.07 & 2,3 & $\operatorname{Ly} \alpha$ \\
\hline 18131 & 34.4512749 & -5.1754861 & 25.493 & 5.985 & 5.81 & 0.0 & $\mathrm{C}$ & $<4$ & -1.74 & -21.03 & 0.08 & 2,3 & Break \\
\hline 18915 & 34.2780228 & -5.1713920 & 25.59 & 5.859 & 5.58 & 0.0 & $\mathrm{C}$ & $<1.5$ & -2.62 & -20.69 & 0.39 & 2,3 & Break \\
\hline 19841 & 34.3490982 & -5.1662178 & 26.34 & 6.370 & 6.836 & $5.0 \mathrm{e}-18$ & $\mathrm{~A} / \mathrm{B}$ & 18 & -1.27 & -20.33 & 0.32 & 2,3 & $\operatorname{Ly} \alpha$ \\
\hline 23719 & 34.3104324 & -5.1456208 & 26.79 & 5.198 & 5.683 & $9.9 \mathrm{e}-18$ & A & 64 & -2.36 & -20.03 & 0.17 & 2,3 & $\operatorname{Ly} \alpha$ \\
\hline 23802 & 34.2283478 & -5.1474319 & 26.18 & 6.884 & 6.634 & $1.7 \mathrm{e}-18$ & $\mathrm{~B} / \mathrm{C}$ & 7 & -2.20 & -20.63 & 0.21 & 1,3 & $\operatorname{Ly} \alpha$ \\
\hline 25826 & 34.2333316 & -5.1364809 & 25.45 & 5.928 & 5.83 & 0.0 & $\mathrm{C}$ & $<2$ & -2.10 & -21.25 & 0.26 & 2,3 & Break \\
\hline 28306 & 34.3560867 & -5.2582278 & 27.13 & 6.007 & 6.142 & $6.5 e-18$ & $\mathrm{~A}$ & 41 & -2.74 & -19.99 & 0.05 & 2,3 & $\operatorname{Ly} \alpha$ \\
\hline 29191 & 34.5253906 & -5.2412128 & 26.48 & 5.879 & 5.943 & $8.8 \mathrm{e}-18$ & $\mathrm{~A} / \mathrm{B}$ & 34 & -3.18 & -20.57 & 0.06 & 2,3 & $\operatorname{Ly} \alpha$ \\
\hline 31124 & 34.2537117 & -5.2067928 & 26.89 & 1.260 & 6.464 & $1.5 \mathrm{e}-18$ & $\mathrm{~B} / \mathrm{C}$ & 8 & -2.06 & -19.98 & 0.15 & 2 & $\operatorname{Ly} \alpha$ \\
\hline 33304 & 34.5186958 & -5.1694598 & 26.26 & 5.866 & 6.033 & $3.5 \mathrm{e}-18$ & $\mathrm{~B}$ & 16 & -2.14 & -20.44 & 0.08 & 2,3 & $\operatorname{Ly} \alpha$ \\
\hline
\end{tabular}

Notes. ${ }^{(a)}$ This galaxy has [CII] $158 \mu \mathrm{m}$ detection published in Pentericci et al. (2016).

Table 4. Properties of confirmed galaxies in the CANDELS COSMOS field.

\begin{tabular}{|c|c|c|c|c|c|c|c|c|c|c|c|c|c|}
\hline ID & $\begin{array}{c}\text { RA } \\
\text { J2000 }\end{array}$ & $\begin{array}{c}\text { Dec } \\
\text { J2000 }\end{array}$ & $H_{160}$ & zphot & zspec & $\begin{array}{c}\text { Flux } \\
\mathrm{erg}^{-1} \mathrm{~s}^{-1} \mathrm{~cm}^{2} \\
\end{array}$ & $\mathrm{QF}$ & $\begin{array}{c}\mathrm{EW}_{\mathrm{Ly \alpha}} \\
\AA\end{array}$ & $\beta$ & $M_{\mathrm{UV}}$ & $\begin{array}{l}r_{\mathrm{e}} \\
\prime \prime\end{array}$ & Sel & Comm. \\
\hline 6822 & 150.177171 & 2.261854 & 25.79 & 0.848 & 5.131 & 0.0 & $\mathrm{~B}$ & $<19$ & -1.69 & -20.50 & 0.23 & 2 & Ly $\alpha$, Break \\
\hline 7499 & 150.089164 & 2.26949 & 26.29 & 5.737 & 5.858 & $6.1 \mathrm{e}-18$ & $\mathrm{~A} / \mathrm{B}$ & 19 & -1.30 & -20.33 & 0.09 & 2,3 & $\operatorname{Ly} \alpha$ \\
\hline 7692 & 150.107757 & 2.271918 & 26.04 & 5.634 & 6.046 & $7.0 \mathrm{e}-18$ & A & 24 & -0.74 & -20.20 & 0.19 & 2,3 & $\operatorname{Ly} \alpha$ \\
\hline 8118 & 150.154944 & 2.277192 & 26.07 & 1.24 & 5.731 & $2.7 \mathrm{e}-18$ & $\mathrm{~A} / \mathrm{B}$ & 7 & -1.71 & -20.42 & 0.14 & 2 & Ly $\alpha$ \\
\hline 8474 & 150.106609 & 2.281278 & 25.00 & 6.079 & 0.661 & - & A & - & - & - & 0.55 & 2,3 & $\mathrm{H} \beta$ OIII \\
\hline 10699 & 150.118281 & 2.307277 & 26.61 & 5.778 & 5.835 & $2.5 \mathrm{e}-18$ & $\mathrm{C}$ & 9 & -2.79 & -20.33 & 0.02 & 2,3 & $\operatorname{Ly} \alpha$ \\
\hline 12306 & 150.127459 & 2.326425 & 24.31 & 6.197 & 5.908 & $2.8 \mathrm{e}-18$ & $\mathrm{~A} / \mathrm{B}$ & 3 & -1.70 & -22.26 & 0.45 & 2,3 & $\operatorname{Ly} \alpha$ \\
\hline $13679^{a}$ & 150.099037 & 2.343627 & 25.43 & 6.525 & 7.145 & $9.2 \mathrm{e}-18$ & $\mathrm{~A} / \mathrm{B}$ & 15 & -1.54 & -21.46 & 0.01 & 1,3 & $\operatorname{Ly} \alpha$ \\
\hline 18472 & 150.126605 & 2.401444 & 25.63 & 5.899 & 5.86 & 0.0 & $\mathrm{C}$ & $<5$ & -1.94 & -21.03 & 0.02 & 2,3 & Break \\
\hline 20521 & 150.139594 & 2.426985 & 25.69 & 6.46 & 6.360 & $6.5 \mathrm{e}-18$ & B & 10 & -2.17 & -21.13 & 0.17 & 2,3 & $\operatorname{Ly} \alpha$ \\
\hline 21151 & 150.165997 & 2.435793 & 26.23 & 1.522 & 7.040 & $1.65 \mathrm{e}-17$ & $\mathrm{~A}$ & 65 & -1.25 & -20.57 & 0.05 & 1 & Ly $\alpha$ \\
\hline 21411 & 150.183018 & 2.439205 & 25.77 & 6.066 & 6.221 & $3.8 \mathrm{e}-17$ & A & 90 & -1.40 & -20.77 & 1.51 & 2,3 & Ly $\alpha$ \\
\hline 22592 & 150.196765 & 2.454824 & 24.15 & 6.39 & 5.60 & 0.0 & $\mathrm{C}$ & $<1.2$ & -2.28 & -22.54 & 0.02 & 2 & Break \\
\hline $24108^{a}$ & 150.197222 & 2.478651 & 25.14 & 1.297 & 6.629 & $2.0 e-17$ & A & 27 & -1.76 & -21.67 & 0.27 & 2 & $\operatorname{Ly} \alpha$ \\
\hline 25022 & 150.191343 & 2.492327 & 25.99 & 5.847 & 5.62 & 0.0 & $\mathrm{C}$ & $<2$ & -1.56 & -20.37 & 0.11 & 3 & Break \\
\hline 26366 & 150.161146 & 2.511134 & 25.84 & 6.013 & 6.25 & 0.0 & $\mathrm{C}$ & $<2$ & -0.89 & -20.45 & 0.04 & 3 & Break \\
\hline 30549 & 150.162535 & 2.234355 & 26.65 & 1.19 & 6.730 & $3.0 e-18$ & $\mathrm{~B} / \mathrm{C}$ & 16 & -1.76 & -19.96 & 0.05 & 1 & Ly $\alpha$ \\
\hline 36393 & 150.118121 & 2.451689 & 26.17 & 6.522 & 5.377 & $4.0 \mathrm{e}-17$ & $\mathrm{~B}$ & 12 & -2.33 & -20.146 & 0.30 & 2,3 & $\operatorname{Ly} \alpha$ \\
\hline
\end{tabular}

Notes. ${ }^{(a)}$ These galaxies have [CII] $158 \mu$ m detections published in Pentericci et al. (2016).

our new results indicate both a possible flattening in the evolution with redshift of the Ly $\alpha$ fraction between $z \sim 5$ and $z \sim 6$, instead of a steady increase up to $z \sim 6$, and that the downturn between $z \sim 6$ and $z \sim 7$ is somewhat less strong than previously reported, especially for large values of REW (e.g., LP14). Assuming that the visibility of the Ly $\alpha$ line depends only on the IGM neutral hydrogen content, this could mean that the increase of this quantity might be less rapid and could continue also at $z<6$. This would also be in agreement with some recent measurements from quasar proximity zones, which are consistent with a shallower evolution of the IGM neutral fraction during the epoch of reionization (Eilers et al. 2017). Similarly, the recent discovery of an extreme Ly $\alpha$ trough below redshift 6 (Becker et al. 2015) is consistent with the scenario where reionization is still ongoing at $z \sim 6$, and is fully completed only by $z \sim 5.5$.

\subsection{The shape of the Lyo emission}

The shape of the $\operatorname{Ly} \alpha$ line is potentially another tool to probe the reionization epoch: its width and the asymmetric properties are expected to change in a partially neutral IGM (e.g., Dijkstra et al. 2007). With our medium-resolution spectra $(R=1390)$ we 


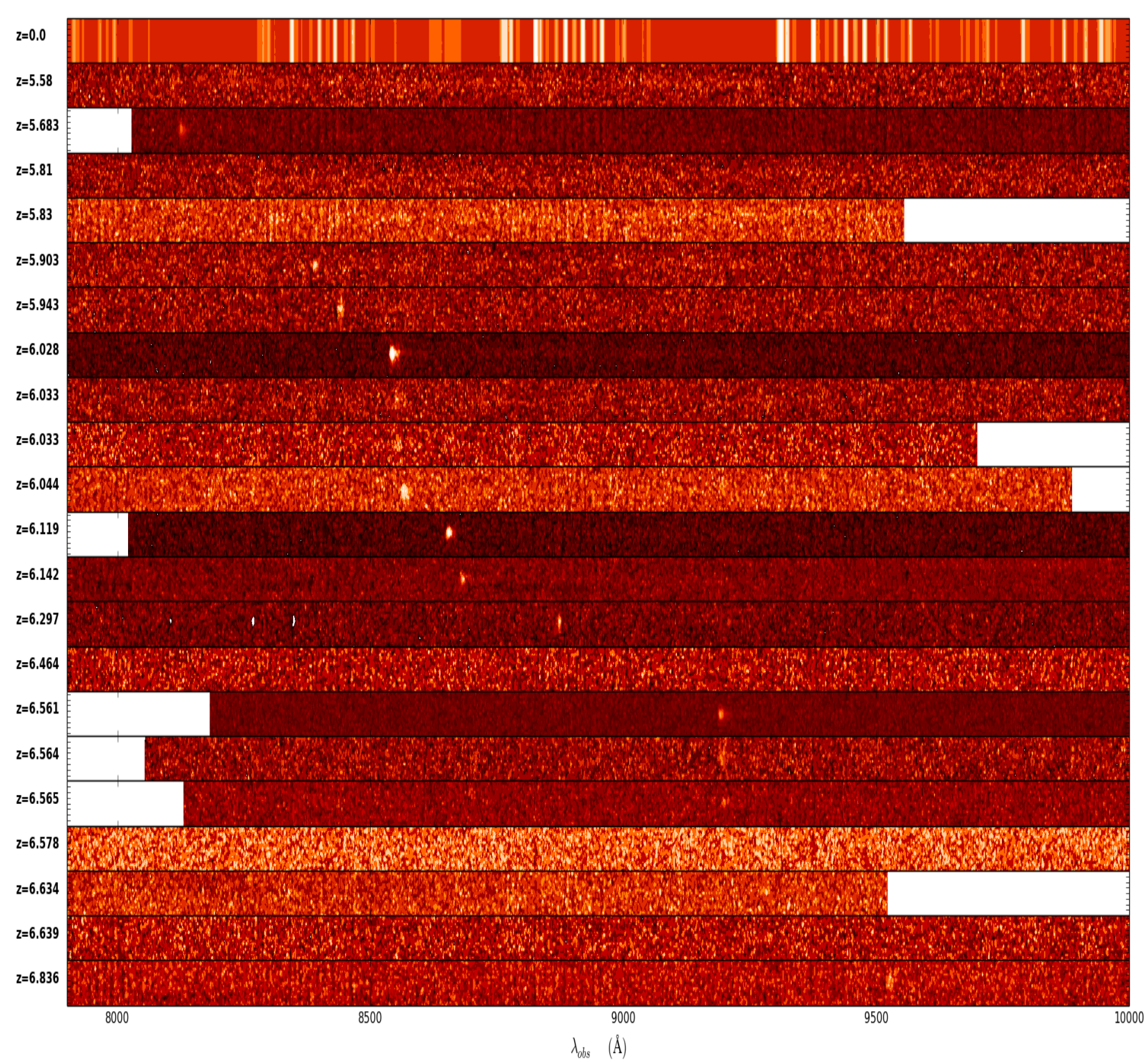

Fig. 11. 2D spectra of the 21 newly confirmed galaxies in the UDS field from top to bottom in order of increasing redshifts registered to the same observed wavelength range. We plot here the maps of the $\mathrm{S} / \mathrm{N}$ which were obtained from the sky subtracted data dividing them by the map of the noise spectrum. The top five galaxies show a faint continuum. The bottom panel represents the sky spectrum.

can investigate the evolution of the line profile of Ly $\alpha$-emitting galaxies between $z \sim 6$ and $z \sim 7$. Because the $\mathrm{S} / \mathrm{N}$ of individual spectra is mostly too low for an accurate spectral fit, we produced stacked spectra of all galaxies in the two sredshift intervals investigated: we considered 19 galaxies at $z>6.5$ (the ones from CANDELSz7, and previous spectra from V11, LP11, and LP14) and 50 galaxies at $5.5<z<6.5$ (same references). To produce the stacks, we first shifted each 1D spectrum to its rest-frame using the redshift evaluated from the peak of the Ly $\alpha$ line. Subsequently, using a linear interpolation, we resampled each spectrum to the same grid that goes from 1100 to $1250 \AA$ with a step of $1.6 \AA /\left(1+z_{\text {median }}\right)$ where $1.6 \AA$ is the nominal resolution of the observed-frame spectra. To take into account the noise of each spectrum, we computed the stack as a weighted average of the spectra, using the $\mathrm{S} / \mathrm{N}$ of the Ly $\alpha$ lines as weights. These have been evaluated by dividing the total flux of the Ly $\alpha$ line by the noise of the spectrum, estimated as the dispersion around the mean value in the wavelength range $1225-1250 \AA$. We chose this relatively small inter- val because it is the only range that is present in all spectra and is not affected by the flux of the $\operatorname{Ly} \alpha$ emission line. We did not normalize the spectra during the stacking procedure, since they have basically the same level of the continuum. The final stacks for the two redshift bins are presented in Fig. 10. We can see that the Ly $\alpha$ emission is in both cases very asymmetric with extended red wings. The blue side of the line is completely compatible with the instrumental resolution for the $z \sim 7$ stack, while it is broader for the $z \sim 6$ stack. From the figure we can also see that there is a faint continuum in the lowerredshift stack red-ward of the emission line which is not present in the higher-redshift one, where the flux is consistent with zero.

Since the $z \sim 6$ sample is much more numerous than the $z \sim 7$ one, part of the broadening could in principle be due to the imperfect alignment of the lines, that is, errors in the line-center determination for the low-S/N lines. To check if this is the case, we further divided the lower-redshift sample into two bins, one containing galaxies in the interval $z=[5.5-6]$, and the other 


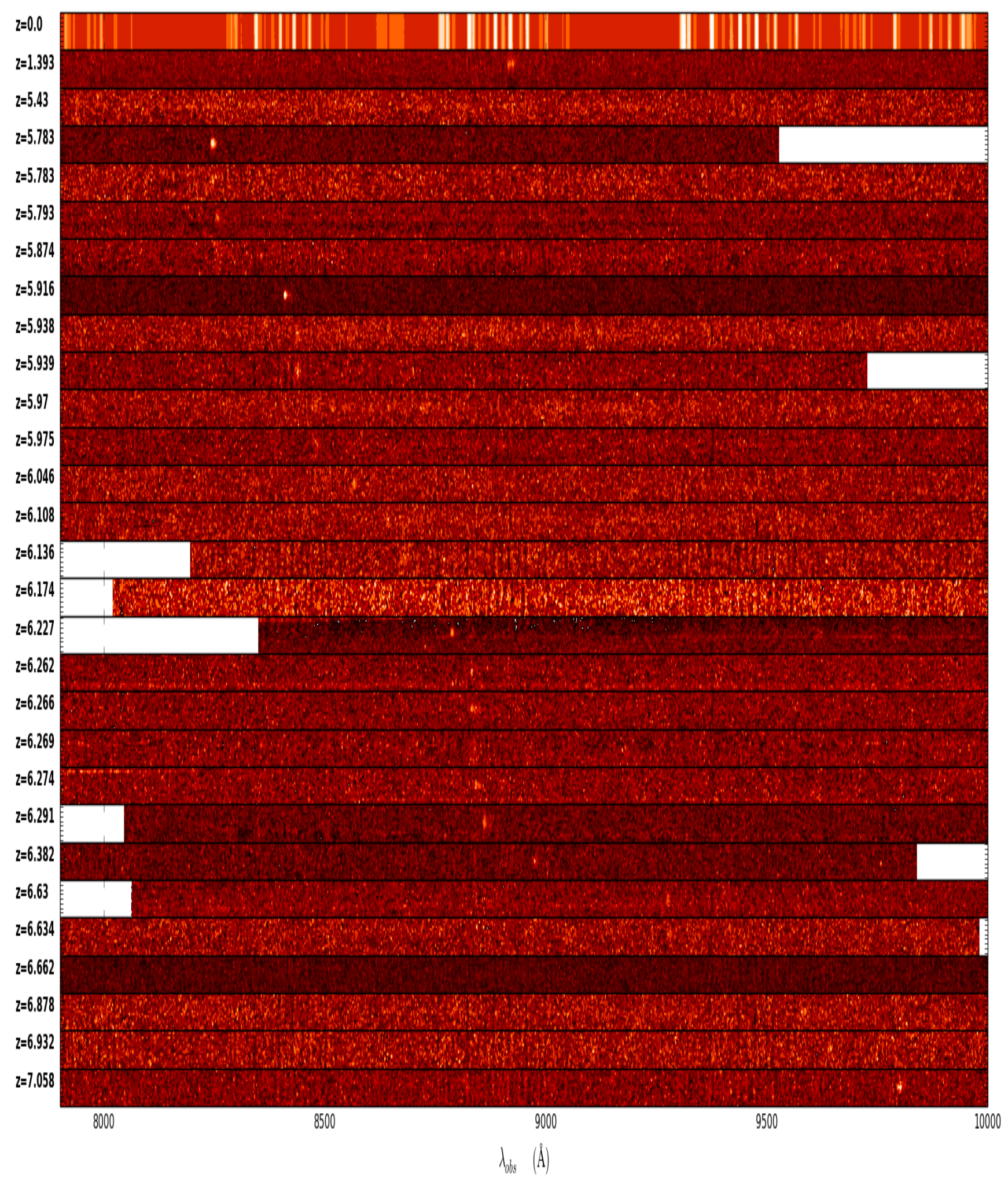

Fig. 12. 2D spectra of the 28 newly confirmed galaxies in the GOODS-South field from top to bottom in order of increasing redshift, registered to the same observed wavelength range. We plot here the maps of the $\mathrm{S} / \mathrm{N}$ which were obtained from the sky subtracted data, by dividing them by the map of the noise spectrum. The top panel represents the sky spectrum.

containing galaxies in the interval $z=[6-6.5]$. In the inset of Fig. 10 we show that the two lower-redshift stacks are consistent with each other and that the line is in both cases broader that at $z \sim 7$, so the result is not due to the sample size.

We determine the FWHM of the stacked Ly $\alpha$ line: we first fit a simple Gaussian profile to the stacks and de-convolve the val- ues by the resolution of the FORS 2 spectra. The velocity widths we obtain are $300 \pm 30 \mathrm{~km} \mathrm{~s}^{-1}$ and $220 \pm 30 \mathrm{~km} \mathrm{~s}^{-1}$ at $z \sim 6$ and $z \sim 7$, respectively, confirming that the line is slightly broader (at $2 \sigma$ significance) in the lower redshift stack. Since the lines are very asymmetric, the Gaussian fit is not a good representation of their shape. If we measure the FWHM directly from 
Table 5. Properties of confirmed galaxies in the CANDELS GOODS-South field.

\begin{tabular}{|c|c|c|c|c|c|c|c|c|c|c|c|c|c|}
\hline ID & $\begin{array}{c}\text { RA } \\
\text { J2000 } \\
\end{array}$ & $\begin{array}{c}\text { Dec } \\
\text { J2000 }\end{array}$ & $H_{160}$ & zphot & zspec & $\begin{array}{c}\text { Flux } \\
\operatorname{erg}^{-1} \mathrm{~s}^{-1} \mathrm{~cm}^{2} \\
\end{array}$ & QF & $\begin{array}{c}\mathrm{EW}_{\mathrm{Ly} \alpha} \\
\AA\end{array}$ & $\beta$ & $M_{\mathrm{UV}}$ & $\begin{array}{l}r_{\mathrm{e}} \\
1\end{array}$ & Sel & Comm. \\
\hline 10219 & 53.2020264 & -27.8163528 & 26.14 & 5.997 & 6.136 & $4.0 e-18$ & B & 14 & -1.57 & -20.56 & 0.22 & 2,3 & $\operatorname{Ly} \alpha$ \\
\hline 11464 & 53.1174545 & -27.8051872 & 26.04 & 5.994 & 5.939 & 7.0e-18 & B & 22 & -1.59 & -20.53 & 0.14 & 2,3 & $\operatorname{Ly} \alpha$ \\
\hline 12881 & 53.0694046 & -27.7943935 & 26.11 & 5.9360 & 5.874 & $2.0 e-18$ & $\mathrm{~B} / \mathrm{C}$ & 5 & -2.22 & -20.70 & 0.37 & 2,3 & $\operatorname{Ly} \alpha$ \\
\hline 13065 & 53.1438484 & -27.7930164 & 28.21 & 6.001 & 6.932 & $2.2 \mathrm{e}-18$ & B & 42 & -1.90 & -18.85 & 0.02 & 2,3 & $\operatorname{Ly} \alpha$ \\
\hline 14439 & 53.1248894 & -27.7841072 & 27.18 & 5.680 & 5.783 & $1.0 e-17$ & A & 50 & -2.86 & -19.93 & 0.15 & 2,3 & $\operatorname{Ly} \alpha$ \\
\hline 15178 & 53.0558853 & -27.7795563 & 26.78 & 6.018 & 6.266 & $9.1 \mathrm{e}-18$ & $\mathrm{~A} / \mathrm{B}$ & 48 & -1.58 & -19.88 & 0.09 & 2,3 & $\operatorname{Ly} \alpha$ \\
\hline 15404 & 53.0338783 & -27.7780075 & 26.70 & 6.706 & 6.291 & $9.0 \mathrm{e}-18$ & A & 44 & -1.30 & -19.94 & 0.09 & 2,3 & $\operatorname{Ly} \alpha$ \\
\hline 15951 & 53.1050529 & -27.7740688 & 26.91 & 6.997 & 6.634 & $2.0 e-18$ & B & 17 & -2.03 & -19.98 & 0.08 & 1,2 & $\operatorname{Ly} \alpha$ \\
\hline 16024 & 53.0731506 & -27.773634 & 25.45 & 5.805 & 5.43 & 0.0 & $\mathrm{C}$ & $<1.6$ & -1.33 & -20.82 & 0.22 & 2,3 & Break \\
\hline 17692 & 53.1627693 & -27.7607594 & 28.09 & 5.574 & 5.916 & $2.25 e-17$ & A & 153 & -2.44 & -18.93 & 0.04 & 2,3 & $\operatorname{Ly} \alpha$ \\
\hline 20698 & 53.2031670 & -27.7337036 & 25.99 & 6.168 & 6.174 & $1.5 \mathrm{e}-18$ & $\mathrm{~B} / \mathrm{C}$ & 4 & -1.45 & -20.75 & 0.18 & 2,3 & $\operatorname{Ly} \alpha$ \\
\hline 22647 & 53.0666809 & -27.7170753 & 25.91 & 1.246 & 5.783 & 0.0 & $\mathrm{~B} / \mathrm{C}$ & $<11$ & -1.77 & -20.78 & 0.92 & 2,3 & $\operatorname{Ly} \alpha$ \\
\hline 26628 & 53.0707664 & -27.7066383 & 26.58 & 5.949 & 5.975 & $2.2 \mathrm{e}-18$ & B & 9 & -2.47 & -20.30 & 0.29 & 2,3 & $\operatorname{Ly} \alpha$ \\
\hline 31131 & 53.1255341 & -27.7866764 & 26.80 & 6.00 & 6.382 & $3.1 \mathrm{e}-18$ & B & 26 & -1.0 & -19.89 & 0.42 & 2,3 & $\operatorname{Ly} \alpha$ \\
\hline 31144 & 53.0830650 & -27.7862740 & 27.31 & 1.306 & 6.269 & $6.0 \mathrm{e}-18$ & $\mathrm{~B} / \mathrm{C}$ & 65 & -1.61 & -19.28 & 0.20 & 1 & $\operatorname{Ly} \alpha$ \\
\hline 32252 & 53.0951385 & -27.7605419 & 27.55 & 6.183 & 6.262 & 0.0 & $\mathrm{~B} / \mathrm{C}$ & $<23$ & -2.99 & -19.64 & 0.08 & 2,3 & $\operatorname{Ly} \alpha$ \\
\hline 33418 & 53.1944962 & -27.7263489 & 27.40 & 6.373 & 7.058 & $1.2 \mathrm{e}-17$ & A & 110 & -2.2 & -19.54 & 0.04 & 1,2 & $\operatorname{Ly} \alpha$ \\
\hline 33477 & 53.0641861 & -27.7246075 & 27.51 & 6.379 & 6.274 & $6.2 \mathrm{e}-18$ & $\mathrm{~A} / \mathrm{B}$ & 64 & -2.18 & -19.26 & 0.72 & 3 & $\operatorname{Ly} \alpha$ \\
\hline 34061 & 53.0461273 & -27.7082958 & 26.53 & 5.68 & 6.227 & $6.8 \mathrm{e}-18$ & A & 36 & -0.35 & -19.79 & $0.18^{b}$ & 2,3 & $\operatorname{Ly} \alpha$ \\
\hline $\mathrm{H} 2525^{a}$ & 53.145531 & -27.783724 & 27.98 & 6.78 & 6.878 & $2.0 \mathrm{e}-18$ & $\mathrm{~B} / \mathrm{C}$ & 18 & -1.98 & -19.66 & - & 1,2 & $\operatorname{Ly} \alpha$ \\
\hline 13184 & 53.1519318 & -27.7923527 & 27.46 & 1.264 & 6.662 & $5.0 \mathrm{e}-18$ & $\mathrm{~A} / \mathrm{B}$ & 81 & -0.35 & -19.05 & 0.21 & 1 & $\operatorname{Ly} \alpha$ \\
\hline 15443 & 53.1519432 & -27.7781773 & 25.73 & 5.933 & 5.938 & $3.6 e-18$ & A & 7 & -1.89 & -20.98 & 0.15 & 2,3 & $\operatorname{Ly} \alpha$ \\
\hline 16371 & 53.1595078 & -27.7714462 & 26.35 & 6.123 & 6.108 & $3.0 \mathrm{e}-18$ & B & 11 & -2.01 & -20.43 & 0.04 & 2,3 & $\operatorname{Ly} \alpha$ \\
\hline 18310 & 53.1419334 & -27.7551537 & 27.26 & 5.867 & 6.046 & $3.0 \mathrm{e}-18$ & B & 26 & -1.66 & -19.34 & 0.03 & 2,3 & $\operatorname{Ly} \alpha$ \\
\hline 22194 & 53.1161804 & -27.7210217 & 26.70 & 5.541 & 5.793 & $5.0 \mathrm{e}-18$ & B & 24 & -2.00 & -19.90 & 0.35 & 2,3 & Ly $\alpha$ \\
\hline 26560 & 53.1581459 & -27.7021046 & 24.55 & 6.22 & 5.97 & 0.0 & $\mathrm{C}$ & $<1.3$ & -1.38 & -21.99 & 0.24 & 2,3 & Break \\
\hline 31759 & 53.1348877 & -27.7726326 & 27.47 & 6.174 & 1.393 & $1.27 \mathrm{e}-17$ & A & - & - & - & 0.02 & 2,3 & [OII] \\
\hline 31891 & 53.1742516 & -27.7697887 & 28.75 & 6.386 & 6.630 & $2.0 \mathrm{e}-18$ & A & 73 & -1.85 & -18.08 & 0.03 & 1,3 & $\operatorname{Ly} \alpha$ \\
\hline
\end{tabular}

Notes. ${ }^{(a)}$ This galaxy does not have a CANDELS ID, since it is detected in the hot-mode only but it is located within the Kron radius of a cold source and therefore excluded from the final catalog (Guo et al. 2013). ${ }^{(b)}$ The $r_{\mathrm{e}}$ reported is measured in $Y_{098}$ filter since the $J_{125}$ is contaminated.

Table 6. Fitted parameters for the Ly $\alpha$ emission line in the two stacks.

\begin{tabular}{cccccc}
\hline \hline$\langle z\rangle$ & $N$ & $\begin{array}{c}\left\langle L_{\mathrm{Ly} \alpha}\right\rangle \\
\mathrm{erg} \mathrm{s}^{-1}\end{array}$ & $\left\langle M_{\mathrm{UV}}\right\rangle$ & $\begin{array}{c}F W H M \\
\mathrm{~km} \mathrm{~s}^{-1}\end{array}$ & $S_{\mathrm{W}}$ \\
\hline 6.0 & 52 & $2.5 \times 10^{42}$ & -20.53 & $300 \pm 30$ & $15.8 \pm 8$ \\
6.9 & 19 & $2.7 \times 10^{42}$ & -20.62 & $220 \pm 25$ & $25 \pm 10$ \\
\hline
\end{tabular}

Notes. $\langle z\rangle$ median redshift of the sample; $N$ total number of objects in each sample; $\left\langle L_{\mathrm{Ly} \alpha}\right\rangle$ median Ly $\alpha$ luminosity of the sample; $\left\langle M_{\mathrm{UV}}\right\rangle$ median $M_{\mathrm{UV}}$; FWHM of the stacked lines from the Gaussian fit; $S_{\mathrm{W}}$ weighted skewness parameter of the stacked lines.

the line, that is, with no fit, we find just slightly smaller values, $290 \pm 25 \mathrm{~km} \mathrm{~s}^{-1}$ and $215 \pm 20 \mathrm{~km} \mathrm{~s}^{-1}$, respectively, also after deconvolution with the instrumental resolution. In all cases the uncertainties at $68 \%$ level are derived using the bootstrapping statistics, by creating 100 realizations of the stacks and randomly extracting $N$ galaxies with replacement. To quantify the asymmetry of the Ly $\alpha$ line, Shimasaku et al. (2006) introduced the weighted skewness $S_{\mathrm{W}}$ which is the skewness (or third moment) of the line multiplied by $\left(\lambda_{10, \mathrm{r}}-\lambda_{10, \mathrm{~b}}\right)$ where $\lambda_{10, \mathrm{r}}$ and $\lambda_{10, \mathrm{~b}}$ are the wavelengths where the flux drops to $10 \%$ of its peak value at the red and blue sides of the emission, respectively. Since the Ly $\alpha$ emission of high-redshift galaxies tends to be wider than other emission lines of nearby galaxies in the observed frame, this factor enhances the difference between Ly $\alpha$ and other lines. Typically, large positive $S_{\mathrm{W}}$ values are found for high-redshift
Ly $\alpha$ while the $[\mathrm{O} \mathrm{III}]$ and $\mathrm{H} \alpha$ lines are nearly symmetric, that is, $S_{\mathrm{W}}=0$, and [O II] emitters are also expected to have small negative skewness. Therefore this parameter can help distinguish Ly $\alpha$ from other emission lines. Usually the Ly $\alpha$ has $S_{\mathrm{W}}>3$ and this has been used as a threshold to distinguish this line from others in low-resolution spectra. We evaluated the skewness for the two stacked spectra and the results are $S_{\mathrm{W}}=15.8 \pm 8$ and $S_{\mathrm{W}}=25 \pm 10$ for the $z \sim 6$ and $z \sim 7$ samples, respectively, with the errors at $68 \%$ evaluated using the bootstrapping statistics as above. The high-redshift line is slightly more asymmetric ( $1 \sigma$ difference) that the low-redshift one. For comparison, similar stacks made by Ouchi et al. (2010) on samples of LAEs, with 19 objects at $z=6.6$ and 11 at $z=5.7$ having Ly $\alpha$ luminosities in the same range as our samples $\left(L=10^{42}-10^{43} \mathrm{erg} \mathrm{s}^{-1}\right)$, resulted in FWHM velocity widths of $270 \pm 16 \mathrm{~km} \mathrm{~s}^{-1}$ and $265 \pm 37 \mathrm{~km} \mathrm{~s}^{-1}$ at $z=6.6$ and 5.7, respectively, with no evidence for evolution. Similarly, Vivian et al. (2015) found no evidence of evolution in Ly $\alpha$ asymmetry or axial ratio with look-back time in high-redshift LBGs, although their sample contained only four galaxies at $z>6$.

As extensively discussed by Dijkstra et al. (2007) the observed shape of the Ly $\alpha$ line depends on many factors related to the galaxies' properties, including the star formation rate, the intrinsic width (related to the velocity dispersion of the systems), and the systemic velocity of the lines. For example, galaxies with large star formation rates tend to have more symmetric lines. In our samples, galaxies span a very similar $M_{\mathrm{UV}}$ range (approximately between -18.5 and -22 ) and have similar median Ly $\alpha$ luminosities although the $z \sim 6$ sample extends to slightly higher 


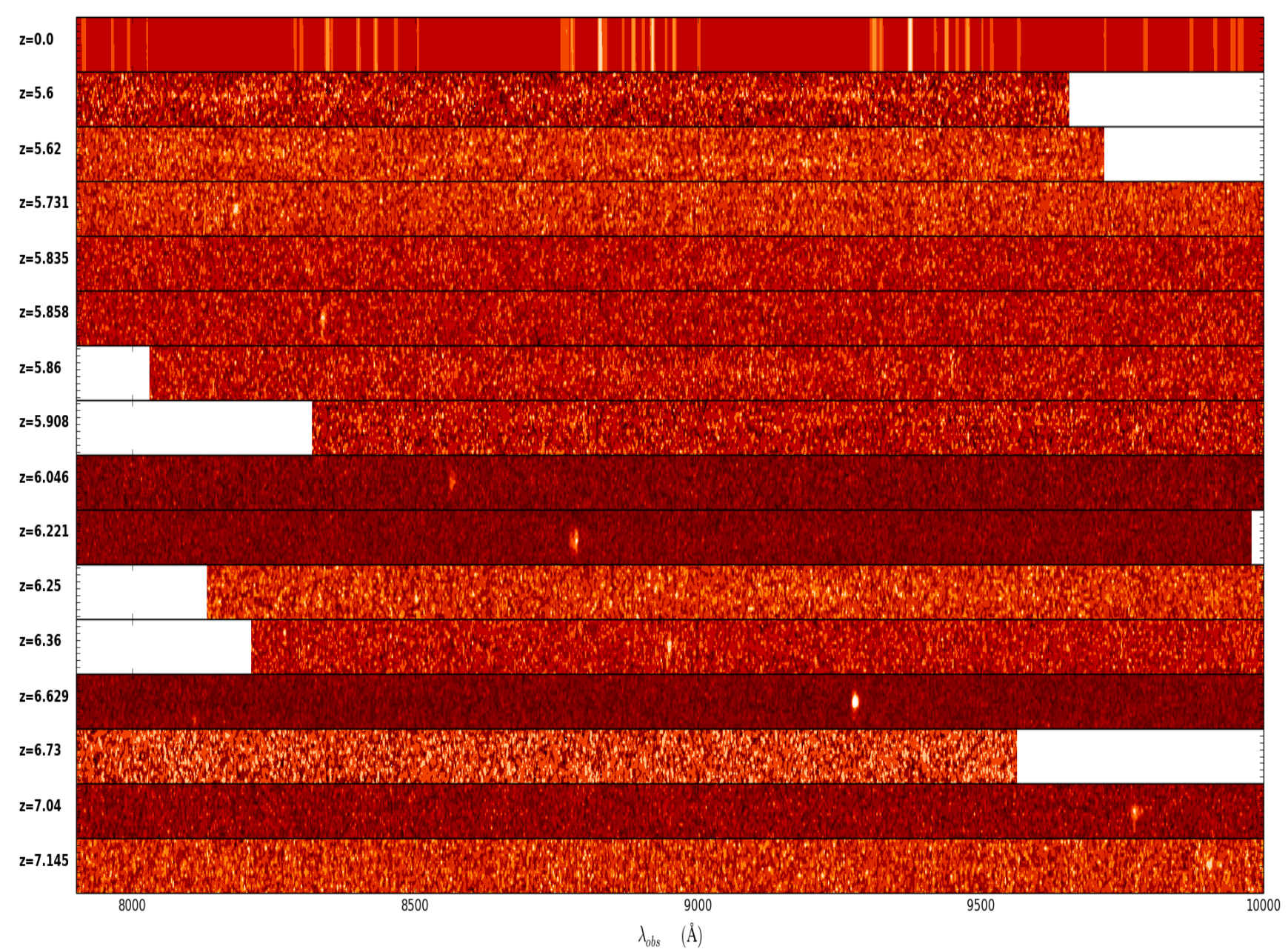

Fig. 13. 2D spectra of 15 newly confirmed galaxies in the COSMOS field, from top to bottom in order of increasing redshifts, registered to the same observed wavelength range. We plot here the maps of the $\mathrm{S} / \mathrm{N}$, which were obtained from the sky subtracted data by dividing them by the map of the noise spectrum. The top panel represents the sky spectrum.

and slightly lower luminosities than the higher-redshift one (see Table 6 for median values). Therefore the small differences in the observed shape of the $\operatorname{Ly} \alpha$ profile, particularly at the blue side, might be due instead to the impact of the IGM. Our results are in qualitative agreement with the simulations by Laursen et al. (2011), who showed that at $z \sim 6$ in some cases an appreciable fraction of the blue wing of the $\operatorname{Ly} \alpha$ line can still be transmitted through the IGM, especially for more massive galaxies $\left(M \geq 1.5 \times 10^{10} M_{\odot}\right.$ and $\left.L_{\mathrm{Ly} \alpha} \geq 10^{42} \mathrm{erg} \mathrm{s}^{-1}\right)$, while at $z>6.5$ the blue wing is always completely erased, regardless of the galaxies' properties.

\section{Summary and conclusions}

We have presented the results of an ESO spectroscopic Large Program aimed at exploring the reionization epoch by observing a large and homogeneous sample of star forming galaxies at redshifts between 5.5 and 7.2 to set firm constraints on the evolution of the Ly $\alpha$ emission fraction at this epoch. Galaxies were selected from the $H_{160}$-band CANDELS catalogs in the GOODS-South, UDS, and COSMOS fields using standard color criteria and/or the official CANDELS photometric redshifts. Spectroscopic observations of 167 high-redshift galaxies were carried out with FORS2, using a medium-resolution red grating. We were able to determine a redshift in 67 objects, mostly from the presence of a single Ly $\alpha$ emission line or, in a few cases, from the detection of continuum flux with a sharp drop that we interpret as the Lyman break; two galaxies are lowredshift interlopers. Overall, the success rate for the identification of the high-redshift targets, for which data could be reduced in a satisfactory way, is $40 \%$. Our sample substantially increases the number of sources with secure spectroscopic redshifts in the CANDELS fields, especially at $z>6.5$, including three new galaxies at $z>7$.

With the newly confirmed galaxies, as well as previous spectroscopic redshifts available in the same fields, we evaluated the accuracy of the CANDELS photometric redshifts at $z \geq 6$. We found that the fraction of catastrophic outliers is $14 \%$, which is more than three times higher than for the lower-redshift galaxies in the rest of the CANDELS catalog, where it is only $\sim 3 \%$. After removing the outliers, the rms uncertainty is 0.036 . We also found that photometric redshifts are in general underestimated for galaxies with $H_{160}>27$ and $z>6.8$, probably due to the presence of a strong $\operatorname{Ly} \alpha$ emission line that influences the broad-band photometry, and which is not taken into account in the photo- $z$ models employed.

Using our medium-resolution spectra we have analyzed the average shape of the Ly $\alpha$ line by creating spectral stacks in two redshift bins. We found that at $z>6.5$ the blue side of the Ly $\alpha$ emission line is completely erased and is consistent with 
the spectral resolution, while at lower redshift a fraction of the blue wing is still transmitted. The Ly $\alpha$ emission has a smaller FWHM and is slightly more asymmetric at $z \sim 7$ compared to $z \sim 6$.

Finally, we have evaluated the distribution of the Ly $\alpha$ restframe EW using the new detections as well as the accurate upper limits determined through extensive simulations, for all the objects where no emission line is observed. The fraction of Ly $\alpha$ emitters that we measure at $z=6$ is consistent with previous determinations only for $\mathrm{REW} \leq 20 \AA$, and it is below for larger REW, with a difference that reaches a factor of greater than two at the highest REWs probed. The fraction of Ly $\alpha$ emitters at $z \sim 6$ is actually consistent with the one determined at $z \sim 5$ (e.g., Stark et al. 2010) indicating a possible flattening in the evolution with redshift between $z \sim 5$ and $z \sim 6$ instead of a steady increase up to $z \sim 6$. The frequency of Ly $\alpha$ drops by an average factor of two between $z \sim 6$ and $z \sim 7$ for galaxies with $M_{\mathrm{UV}}>-20.25$. Overall our results might indicate a possibly slower and more extended reionization process, and in a future paper we will use our new data to set more stringent constraints on the models. In particular, it was shown by Kakiichi et al. (2016) that improved constraints can be derived by analyzing the full $M_{\mathrm{UV}}$-dependent redshift evolution of the Ly $\alpha$ fraction of Lyman break galaxies, such that it would be possible to distinguish between the effect of a "bubble" model, where only diffuse $\mathrm{H}$ I outside ionized bubbles is present, and the "web" model, where H I exists only in over-dense self-shielded gas.

Acknowledgements. This work is based on data obtained from ESO programs 190.A-0685 and 088.A-1013 and was supported by PRIN-INAF-2012: "From the re-jonization epoch to the peak of star formation: investigating galaxy mass assembly and evolution". RM acknowledges support by the Science and Technology Facilities Council (STFC) and the ERC Advanced Grant 695671 "QUENCH".

\section{References}

Becker, G. D., Bolton, J. S., Madau, P., et al. 2015, MNRAS, 447, 3402 Bouwens, R. J., Illingworth, G. D., Oesch, P. A., et al. 2014, ApJ, 793, 115 Bouwens, R. J., Illingworth, G. D., Oesch, P. A., et al. 2015, ApJ, 803, 34 Bouwens, R. J., Aravena, M., Decarli, R., et al. 2016, ApJ, 833, 72

Bradač, M., Vanzella, E., Hall, N., et al. 2012, ApJ, 755, L7

Bradač, M., Garcia-Appadoo, D., Huang, K.-H., et al. 2017, ApJ, 836, L2 Brinchmann, J., Inami, H., Bacon, R., et al. 2017, A\&A, 608, A3 Caruana, J., Bunker, A. J., Wilkins, S. M., et al. 2014, MNRAS, 443, 2831 Caruana, J., Wisotzki, L., Herenz, E. C., et al. 2018, MNRAS, 473, 30 Cassata, P., Tasca, L. A. M., Le Fèvre, O., et al. 2015, A\&A, 573, A24 Castellano, M., Fontana, A., Boutsia, K., et al. 2010a, A\&A, 511, A20 Castellano, M., Fontana, A., Paris, D., et al. 2010b, A\&A, 524, A28 Castellano, M., Fontana, A., Grazian, A., et al. 2012, A\&A, 540, A39 Castellano, M., Pentericci, L., Fontana, A., et al. 2017, ApJ, 839, 73 Chardin, J., Haehnelt, M. G., Bosman, S. E. I., \& Puchwein, E. 2018, MNRAS, 473, 765

Curtis-Lake, E., McLure, R. J., Pearce, H. J., et al. 2012, MNRAS, 422, 1425

Dahlen, T., Mobasher, B., Faber, S. M., et al. 2013, ApJ, 775, 93

De Barros, S., Pentericci, L., Vanzella, E., et al. 2017, A\&A, 608, A123

Dijkstra, M., Lidz, A., \& Wyithe, J. S. B. 2007, MNRAS, 377, 1175

Dijkstra, M., Mesinger, A., \& Wyithe, J. S. B. 2011, MNRAS, 414, 2139

Eilers, A.-C., Davies, F. B., Hennawi, J. F., et al. 2017, ApJ, 840, 24
Fan, X., Strauss, M. A., Becker, R. H., et al. 2006, AJ, 132, 117 Finkelstein, S. L., Ryan, R. E., Jr., Papovich, C., et al. 2015, ApJ, 810, 71 Fontana, A., Vanzella, E., Pentericci, L., et al. 2010, ApJ, 725, L205 Fontana, A., Dunlop, J. S., Paris, D., et al. 2014, A\&A, 570, A11 Galametz, A., Grazian, A., Fontana, A., et al. 2013, ApJS, 206, 10 Gehrels, N. 1986, ApJ, 303, 336

Giallongo, E., Grazian, A., Fiore, F., et al. 2015, A\&A, 578, A83 Grazian, A., Castellano, M., Fontana, A., et al. 2012, A\&A, 547, A51 Grogin, N. A., Kocevski, D. D., Faber, S. M., et al. 2011, ApJS, 197, 35 Gronwall, C., Ciardullo, R., Hickey, T., et al. 2007, ApJ, 667, 79 Guaita, L., Gawiser, E., Padilla, N., et al. 2010, ApJ, 714, 255 Guo, Y., Ferguson, H. C., Giavalisco, M., et al. 2013, ApJS, 207, 24 Hall, N., Bradač, M., Gonzalez, A. H., et al. 2012, ApJ, 745, 155 Herenz, E. C., Urrutia, T., Wisotzki, L., et al. 2017, A\&A, 606, A12 Hoag, A., Bradač, M., Trenti, M., et al. 2017, Nat. Astron., 1, 0091 Huang, K.-H., Lemaux, B. C., Schmidt, K. B., et al. 2016, ApJ, 823, L14 Izotov, Y. I., Schaerer, D., Thuan, T. X., et al. 2016, MNRAS, 461, 3683 Jensen, H., Laursen, P., Mellema, G., et al. 2013, MNRAS, 428, 1366 Kakiichi, K., Dijkstra, M., Ciardi, B., \& Graziani, L. 2016, MNRAS, 463, 4019 Koekemoer, A. M., Faber, S. M., Ferguson, H. C., et al. 2011, ApJS, 197, 36 Laursen, P., Sommer-Larsen, J., \& Razoumov, A. O. 2011, ApJ, 728, 52 Le Fèvre, O., Tasca, L. A. M., Cassata, P., et al. 2015, A\&A, 576, A79 Le Fèvre, O., Lemaux, B. C., Nakajima, K., et al. 2017, A\&A, submitted [arXiv:1710.10715]

Lemaux, B. C., Lubin, L. M., Sawicki, M., et al. 2009, ApJ, 700, 20

Lenz, D. D., \& Ayres, T. R. 1992, PASP, 104, 1104

Maseda, M. V., Brinchmann, J., Franx, M., et al. 2017, A\&A, 608, A4

McGreer, I. D., Mesinger, A., \& D’Odorico, V. 2015, MNRAS, 447, 499

Mesinger, A., Aykutalp, A., Vanzella, E., et al. 2015, MNRAS, 446, 566

Nayyeri, H., Cooray, A., Jullo, E., et al. 2017, ApJ, 844, 82

Oke, J. B., \& Gunn, J. E. 1983, ApJ, 266, 713

Ono, Y., Ouchi, M., Mobasher, B., et al. 2012, ApJ, 744, 83

Ouchi, M., Shimasaku, K., Furusawa, H., et al. 2010, ApJ, 723, 869

Oyarzún, G. A., Blanc, G. A., González, V., et al. 2016, ApJ, 821, L14

Pentericci, L., Fontana, A., Vanzella, E., et al. 2011, ApJ, 743, 132

Pentericci, L., Vanzella, E., Fontana, A., et al. 2014, ApJ, 793, 113

Pentericci, L., Carniani, S., Castellano, M., et al. 2016, ApJ, 829, L11

Planck Collaboration Int. XLVII. 2016, A\&A, 596, A108

Santini, P., Ferguson, H. C., Fontana, A., et al. 2015, ApJ, 801, 97

Schenker, M. A., Stark, D. P., Ellis, R. S., et al. 2012, ApJ, 744, 179

Schenker, M. A., Ellis, R. S., Konidaris, N. P., \& Stark, D. P. 2014, ApJ, 795, 20

Schroeder, J., Mesinger, A., \& Haiman, Z. 2013, MNRAS, 428, 3058

Shapley, A. E., Steidel, C. C., Strom, A. L., et al. 2016, ApJ, 826, L24

Shimasaku, K., Kashikawa, N., Doi, M., et al. 2006, PASJ, 58, 313

Smit, R., Bouwens, R. J., Carniani, S., et al. 2018, Nature, 553, 178

Sobacchi, E., \& Mesinger, A. 2015, MNRAS, 453, 1843

Stanway, E. R., Bunker, A. J., Glazebrook, K., et al. 2007, MNRAS, 376, 727

Stark, D. P., Ellis, R. S., Chiu, K., Ouchi, M., \& Bunker, A. 2010, MNRAS, 408, 1628

Stark, D. P., Ellis, R. S., \& Ouchi, M. 2011, ApJ, 728, L2

Stark, D. P., Walth, G., Charlot, S., et al. 2015, MNRAS, 454, 1393

Stark, D. P., Ellis, R. S., Charlot, S., et al. 2017, MNRAS, 464, 469

Tilvi, V., Rhoads, J. E., Hibon, P., et al. 2010, ApJ, 721, 1853

Tilvi, V., Papovich, C., Finkelstein, S. L., et al. 2014, ApJ, 794, 5

Tilvi, V., Pirzkal, N., Malhotra, S., et al. 2016, ApJ, 827, L14

Totani, T., Aoki, K., Hattori, T., et al. 2014, PASJ, 66, 63

Treu, T., Schmidt, K. B., Trenti, M., Bradley, L. D., \& Stiavelli, M. 2013, ApJ, 775, L29

van der Wel, A., Bell, E. F., Häussler, B., et al. 2012, ApJS, 203, 24

Vanzella, E., Cristiani, S., Dickinson, M., et al. 2008, A\&A, 478, 83

Vanzella, E., Giavalisco, M., Dickinson, M., et al. 2009, ApJ, 695, 1163

Vanzella, E., Pentericci, L., Fontana, A., et al. 2011, ApJ, 730, L35

Vanzella, E., Fontana, A., Zitrin, A., et al. 2014, ApJ, 783, L12

Vanzella, E., de Barros, S., Vasei, K., et al. 2016, ApJ, 825, 41

Vivian, U., Hemmati, S., Darvish, B., et al. 2015, ApJ, 815, 57

Watson, D., Christensen, L., Knudsen, K. K., et al. 2015, Nature, 519, 327 